This item was submitted to Loughborough's Research Repository by the author.

Items in Figshare are protected by copyright, with all rights reserved, unless otherwise indicated.

\title{
Absolute and convective instabilities in counter-current gas-liquid film flows
}

PLEASE CITE THE PUBLISHED VERSION

http://dx.doi.org/10.1017/jfm.2014.667

PUBLISHER

(c) Cambridge University Press

VERSION

AM (Accepted Manuscript)

\section{PUBLISHER STATEMENT}

This work is made available according to the conditions of the Creative Commons Attribution-NonCommercialNoDerivatives 4.0 International (CC BY-NC-ND 4.0) licence. Full details of this licence are available at: https://creativecommons.org/licenses/by-nc-nd/4.0/

\section{LICENCE}

CC BY-NC-ND 4.0

\section{REPOSITORY RECORD}

Vellingiri, Rajagopal, Dmitri Tseluiko, and Serafim Kalliadasis. 2014. "Absolute and Convective Instabilities in Counter-current Gas-liquid Film Flows”. Loughborough University. https://hdl.handle.net/2134/18423. 


\title{
Absolute and convective instabilities in counter-current gas-liquid film flows
}

\author{
Rajagopal Vellingiri ${ }^{1}$, Dmitri Tseluiko ${ }^{2}$ \\ and Serafim Kalliadasis ${ }^{1}$ \\ ${ }^{1}$ Department of Chemical Engineering, Imperial College London, London SW7 2AZ, UK \\ ${ }^{2}$ Department of Mathematical Sciences, Loughborough University, Loughborough LE11 3TU, \\ UK
}

(Received ?; revised ?; accepted ?. - To be entered by editorial office)

We consider a thin liquid film flowing down an inclined plate in the presence of a countercurrent turbulent gas. By making appropriate assumptions, Tseluiko \& Kalliadasis (2011) developed low-dimensional non-local models for the liquid problem, namely a long-wave model and a weighted integral-boundary-layer model, that incorporate the effect of the turbulent gas. By utilising these models, along with the Orr-Sommerfeld problem formulated using the full governing equations for the liquid phase and associated boundary conditions, we explore the linear stability of the gas-liquid system. In addition, we devise a generalised methodology to investigate absolute and convective instabilities in the nonlocal equations describing the gas-liquid flow. We observe that at low gas flow rates, the system is convectively unstable with the localised disturbances being convected downwards. As the gas flow rate is increased, the instability becomes absolute and localised disturbances spread across the whole domain. As the gas flow rate is further increased, the system again becomes convectively unstable with the localised disturbances propagating upwards. We find that the upper limit of the absolute instability region is close to the 'flooding' point associated with the appearance of large-amplitude standing waves, as obtained in Tseluiko \& Kalliadasis (2011), and our analysis can therefore be used to predict the onset of flooding. We also find that an increase in the angle of inclination of the channel requires an increased gas flow rate for the onset of absolute instability. We generally find good agreement between the results obtained using the full equations and the reduced models. Moreover, we find that the weighted integral-boundary-layer model generally provides better agreement with the results for the full equations than the longwave model. Such an analysis is important for understanding the ranges of validity of the reduced model equations. In addition, a comparison of our theoretical predictions with the experiments of Zapke \& Kröger (2000a) shows a fairly good agreement. We supplement our stability analysis with time-dependent computations of the linearised weighted integral-boundary-layer model. To provide some insight into the mechanisms of instability, we perform an energy budget analysis.

Key words:

\section{Introduction}

Gas-liquid flows are important in a variety of physical and technological settings, ranging from the shearing of water layer in oceans to the flow of hydrocarbons in oil pipelines. For example, in the transport of high-viscous crude oil through long-distance pipelines 
using a core-annular flow technique, a thin water film is introduced to surround the inner oil core, with the waves on the oil-water interface playing a crucial role in determining the stability of the two liquid layer. Other engineering systems that utilise gas-liquid flows include distillation, absorption and the cooling of electronic devices. An accurate description and fundamental understanding of the spatiotemporal evolution of two-fluid interfaces and the associated wave formation processes, is crucial for the design and optimisation of processes and devices that exploit two-fluid interfaces.

Thin liquid films flowing over inclined plates have been the subject of many studies since the first experiments of Kapitza (1948). This apparently simple system exhibits a rich dynamical behaviour starting with a flat film that undergoes a series of bifurcations eventually ending in spatiotemporal chaos. For small values of the liquid flow rate, the free surface is fundamentally two-dimensional, consisting of single-hump solitary waves that are formed as a result of the primary wave field undergoing a secondary instability. Such solitary waves are observed in a number of theoretical (Chang et al. 1995; Malamataris \& Balakotaiah 2008) and experimental studies (Liu \& Gollub 1994; Vlachogiannis \& Bontozoglou 2001). For extensive reviews of falling liquid film studies, the reader is referred to the monographs by Alekseenko et al. (1994), Chang \& Demekhin (2002) and Kalliadasis et al. (2012).

The addition of a gas layer to the liquid film makes the system dynamics even more complex and interesting. This results in a number of flow regimes such as bubbly flow, slug flow, wavy flow, churn flow and annular flow, to name a few (Azzopardi 2006). Owing to a number of applications and the intriguing underlying physics, gas-liquid flows remain an active topic of research for more than six decades. Hanratty \& Engen (1957) were amongst the first to analyse stratified gas-liquid flows. These authors investigated the co-current flow of air-water system, and showed various wave transitions through their experiments. Craik (1966) studied experimentally thin water films in a horizontal channel, and reported the presence of fast and slow waves, that traveled faster and slower than the interface, respectively. When the gas velocity is increased beyond a particular value, it results in the formation of roll waves at the interface which is a signature of droplet entrainment into the gas stream (Woodmansee \& Hanratty 1969). Demekhin (1981) and Trifonov (2010) analysed the vertical gas-liquid problem by following the quasi-laminar approach of Miles (1957) and Benjamin (1957). Also Trifonov (2010) solved the full Navier-Stokes equations for the liquid problem whereas Demekhin (1981) developed an integral-boundary-layer model. Recently, Ó Náraigh et al. (2011) considered turbulent flow over a liquid layer by developing a single equation model for the interfacial turbulence. By solving the full two-phase flow problem for the horizontal configuration and on comparison with various models such as the simple quasi-laminar model, eddy viscosity model, visco-elastic model and the rapid distortion theory, they concluded that the waviness induced Reynolds stresses do not play a significant role in determining the stability of the interface. Tseluiko \& Kalliadasis (2011) solved the counter-current gas-liquid problem by using an improved version of the quasi-laminar approach of Miles (1957) and Benjamin (1957). However, their analysis of the gas problem was performed using a curvilinear boundary-layer coordinate system, which gives significantly better agreement with experiments, unlike the solution to the gas problem using a Cartesian coordinate system by Demekhin (1981) and Trifonov (2010). Further, Tseluiko \& Kalliadasis (2011) developed non-local long-wave (LW) and weighted integral-boundary-layer (WIBL) models for the liquid problem to investigate the phenomenon of 'flooding', which corresponds to the appearance of large amplitude standing waves. A weakly nonlinear non-local model obtained by assuming small-amplitude waves was analysed in Lin et al. 
(2013). In the co-current version of the problem, the nonlinear wave speed was found to increase with increase in the gas shear and the liquid flow rate (Vellingiri et al. 2013).

Miesen \& Boersma (1995) analysed the stability of a vertical thin liquid film sheared by a co-flowing turbulent gas. By considering the dynamic effects of the gas, they found two modes of instability, namely the interfacial mode and the internal mode. The interfacial mode has a phase speed larger than the maximum velocity in the liquid layer, whereas the phase speed of the internal mode is smaller than the maximum velocity in the liquid film. They also compared their model calculations with experiments, and they tried to link the generation of roll waves with droplet entrainment. In another study, Boomkamp \& Miesen (1996) provided a general classification of instabilities in parallel two-phase flows based on the energy transfer from the primary to the disturbed flow. They classified the existing studies on such instabilities into five different groups, based on the mechanism of energy transfer, which in turn depends on the properties of the flow system, viz. density stratification and orientation, curvature of the velocity profile, viscosity stratification, shear effects or a combination of the last two. Camassa et al. (2012) performed experiments on a vertical core-annular flow system, where a thin liquid film lining the inside of a circular tube is driven by a counter-current turbulent air flow. They also compared their experimental results with a simple long-wave model. Finally, in a recent study, Dietze \& Ruyer-Quil (2013) considered a thin liquid film in the presence of a confined laminar gas for both horizontal and vertical configurations. By making use of the laminar nature of the flow, they developed a model based on the weighted integralboundary-layer approach for both phases, also taking into account the viscous diffusion effects.

In the present study, we are interested in analysing absolute and convective instabilities in a gas-liquid flow system, where a thin liquid film flowing down an inclined wall is in contact with a counter-current turbulent gas flow. According to some of the earlier studies (Shearer \& Davidson 1965; Cetinbudaklar \& Jameson 1969), the flooding point corresponds to the critical gas flow rate where a standing wave is formed on the interface, whose amplitude is much larger than the mean film thickness. We further propose that this critical gas flow rate indeed has a close correspondence with the onset of absolute instability in the system. The absolute instability allows the disturbances to grow at fixed points in space and therefore results in large-amplitude waves that are not necessarily convected downstream or upstream. The theory of absolute and convective instabilities was first introduced in the field of plasma physics (Briggs 1964), and was later developed in fluid dynamics (Huerre \& Monkewitz 1990; Huerre 2000). Absolute and convective instabilities are found in a variety of fluid flow settings such as liquid sheets and jets (Lin 2003), combustion (Juniper 2006) and many other flow processes. These instabilities are also analysed from a more dynamical systems perspective, using e.g. the complex Ginzburg-Landau equation (Tobias et al. 1998; Chaté \& Manneville 1996), a generic model system for a variety of physical systems close to the instability threshold.

In general, the nature of the instability is determined by examining the response of the linear system to a localised disturbance. If the growing disturbance gets advected and moves away from the point of initiation, then the instability is convective. In other words, if the long-time behaviour of the system decays along the ray $x / t=0$, then the system is said to be convectively unstable (see figure 1). On the other hand, if the disturbance grows at every point in space, then the instability is absolute. Convectively unstable flows are also referred to as 'spatial amplifiers', whereas the absolutely unstable flows act like an 'oscillator' (Huerre 2000; Duprat et al. 2007). Gaster (1962) demonstrated that the spatial growth rate could be obtained from the temporal one through an appropriate transformation, but such a transformation is applicable only for near-critical conditions. 

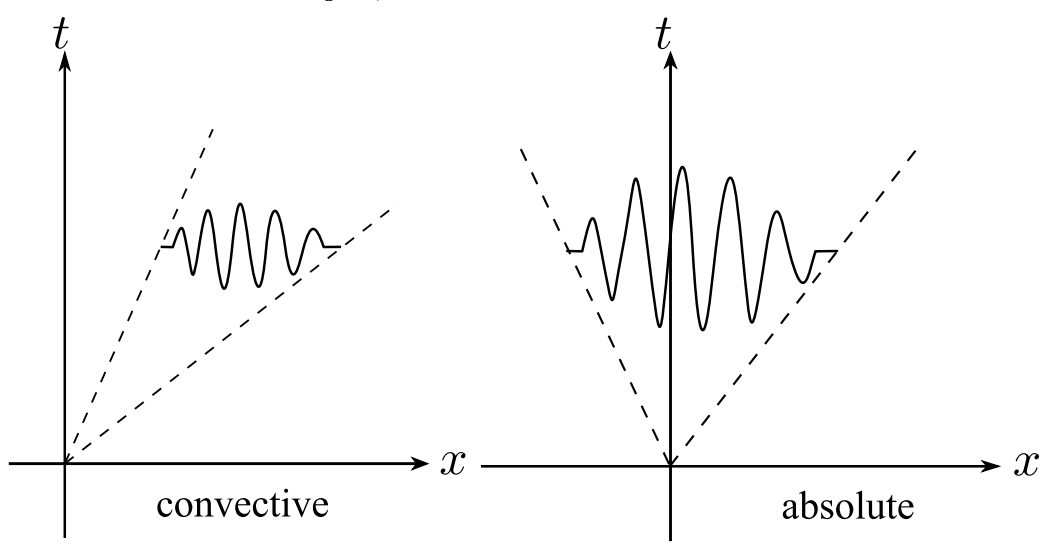

FIGURE 1. Schematic representation of absolute and convective instabilities.

Brevdo et al. (1999) were amongst the first to analyse absolute and convective instabilities in a falling film setting by solving the associated Orr-Sommerfeld (OS) problem. These authors considered two different techniques to analyse absolute and convective instabilities, one based on the exact Briggs collision criterion, and the other based on the tracking of the saddle point which provides the leading-order contribution to the inverse Fourier Laplace transforms. However they found only a convective instability from their numerical studies as expected. Suslov (2006) developed a more complicated algorithm to compute the absolute-convective stability boundary in the given parameter space. Joo \& Davis (1992) analysed instabilities in a falling film using Benney's long-wave evolution equation, and confirmed that the instability is of the convective type. A detailed analysis of the linear stability characteristics of a falling film is given in Kalliadasis et al. (2012). Lin \& Kondic (2010) also considered absolute-convective instabilities in their study on thin film flows on inverted substrates. In a very recent study, Ó Náraigh et al. (2013) analysed absolute and convective instabilities of a horizontal thin liquid film sheared by a fully developed gas flow that could be laminar or turbulent. By combining linear modal and ray analyses, they calculated the regimes of such instabilities and they also found that absolute instability exists for large values of density and viscosity ratios at high Reynolds numbers. To model the turbulent gas flow, they used a simple quasi-laminar assumption where the turbulence contribution enters solely through the base velocity profile thereby ignoring waviness induced Reynolds stresses. Although Ó Náraigh et al. (2011, 2013) found that the waviness induced Reynolds stresses do not play a significant role in the stability of the interface for the parameter values they considered, it should be noted that the simple quasi-laminar assumption does not provide good agreement with experiments - e.g. by Thorsness et al. (1978) - as shown in Tseluiko \& Kalliadasis (2011). Also, the conclusion of Ó Náraigh et al. (2011, 2013) on the impact of waviness induced Reynolds stresses on the interfacial instability seems to be in contradiction with the results presented in Tseluiko \& Kalliadasis (2011). Therefore, an improved version of the quasi-laminar assumption needs to be used, as in Tseluiko \& Kalliadasis (2011). From the modelling point of view, the fact that Ó Náraigh et al. $(2011,2013)$ assume that the turbulence enters only through the base velocity results in a linearised problem that completely ignores the turbulent Reynolds stresses, i.e. it follows that the Reynolds Averaged Navier-Stokes equations are used to obtain the base velocity profile, but the usual Navier-Stokes equations are used for the stability analysis - this is an inconsistency in the sense that the base velocity profile does not satisfy the equations used for the stability 
analysis. In our approach this inconsistency is avoided. From the mathematical point of view, our approach leads to non-local equations, whereas the equations of Ó Náraigh et al. (2013) are local. This means that the standard absolute/convective instability analysis (see e.g. Huerre \& Monkewitz 1990) is not applicable to our situation, and we therefore devise a generalised methodology for analysing absolute/convective instabilities.

Our linear stability analysis, begins with the formulation of a modified OS equation for the vertically falling liquid problem incorporating the effects of counter-current gas flow. As mentioned earlier, in a recent study, Tseluiko \& Kalliadasis (2011) developed a hierarchy of non-local low-dimensional models, namely a long-wave model and a WIBL model to analyse flooding in counter-current gas-liquid flows, focussing mainly on singlehump solitary waves and periodic travelling waves. Their WIBL model is based on the methodology of Ruyer-Quil \& Manneville $(1998,2000)$ for falling liquid films, obtained by combining a long-wave expansion with a polynomial representation of the velocity field, with the integral-boundary-layer approximation and the method of weighted residuals. The advantage with the WIBL model is that it could be applied up to moderate Reynolds numbers. This methodology, appropriately modified, has been applied successfully on falling films in the presence of various complexities. i.e. wall curvature effects (Ruyer-Quil et al. 2008; Ruyer-Quil \& Kalliadasis 2012), thermocapillary Marangoni effects (Trevelyan \& Kalliadasis 2004a,b; Ruyer-Quil et al. 2005; Trevelyan et al. 2007) and solutal Marangoni effect (Pereira \& Kalliadasis 2008). Although it is known that generally WIBL models perform significantly better than Benney-type long-wave models and are valid up to moderate values of the Reynolds number, to truly analyse the range of validity of such long-wave models in any particular setup, it is important to compare their predictions with e.g. experiments or the full equations. The present study is an important step towards such validation. Namely, we perform the linear stability analysis and absolute/convective stability analysis for both the full equations and the reduced models and compare their results.

In $\S 2$ we present the governing equations and the dimensionless parameters. In $\S 3$ we recall the low-dimensional models of Tseluiko \& Kalliadasis (2011). In $\S 4$, we derive the modified OS equation from the Navier-Stokes equations and the associated boundary conditions, and derive the linearised versions for the reduced model equations. By assuming normal mode solutions, we then examine the temporal stability of the system in $\S 5.1$, and in $\$ 5.2$ we perform an energy analysis to elucidate the physical nature of the instability. In $\S 5.3$ we devise a methodology to analyse absolute-convective instabilities. The linear stability results are supplemented by time-dependent computations of the linearised WIBL model in $\$ 5.4$. Finally, we summarise our findings in $\S 6$.

\section{Problem formulation}

Our prototypical system consists of a two-dimensional channel that is inclined at an angle $\theta$ with respect to the horizontal, as depicted in figure 2 . A thin liquid film of density $\rho_{\ell}$ and viscosity $\mu_{\ell}$ flows along the bottom wall of the channel due to gravity. The rest of the channel is occupied with an upflowing gas, which has density $\rho_{g}$ and viscosity $\mu_{g}$. The liquid film is taken to be laminar, whereas the gas flow is considered to be turbulent. We introduce a Cartesian coordinate system $(\tilde{x}, \tilde{y})$ with the $\tilde{x}$-axis pointing along the lower wall and the $\tilde{y}$-axis pointing into the liquid film. Tildes denote dimensional variables.

The governing equations for the liquid problem are the Navier-Stokes equations:

$$
\begin{aligned}
\rho_{\ell}\left(\tilde{\boldsymbol{u}}_{\tilde{t}}+\tilde{\boldsymbol{u}} \cdot \boldsymbol{\nabla} \tilde{\boldsymbol{u}}\right) & =-\nabla \tilde{p}+\mu_{\ell} \nabla^{2} \tilde{\boldsymbol{u}}+\rho_{\ell} \boldsymbol{g}, \\
\nabla \cdot \tilde{\boldsymbol{u}} & =0
\end{aligned}
$$




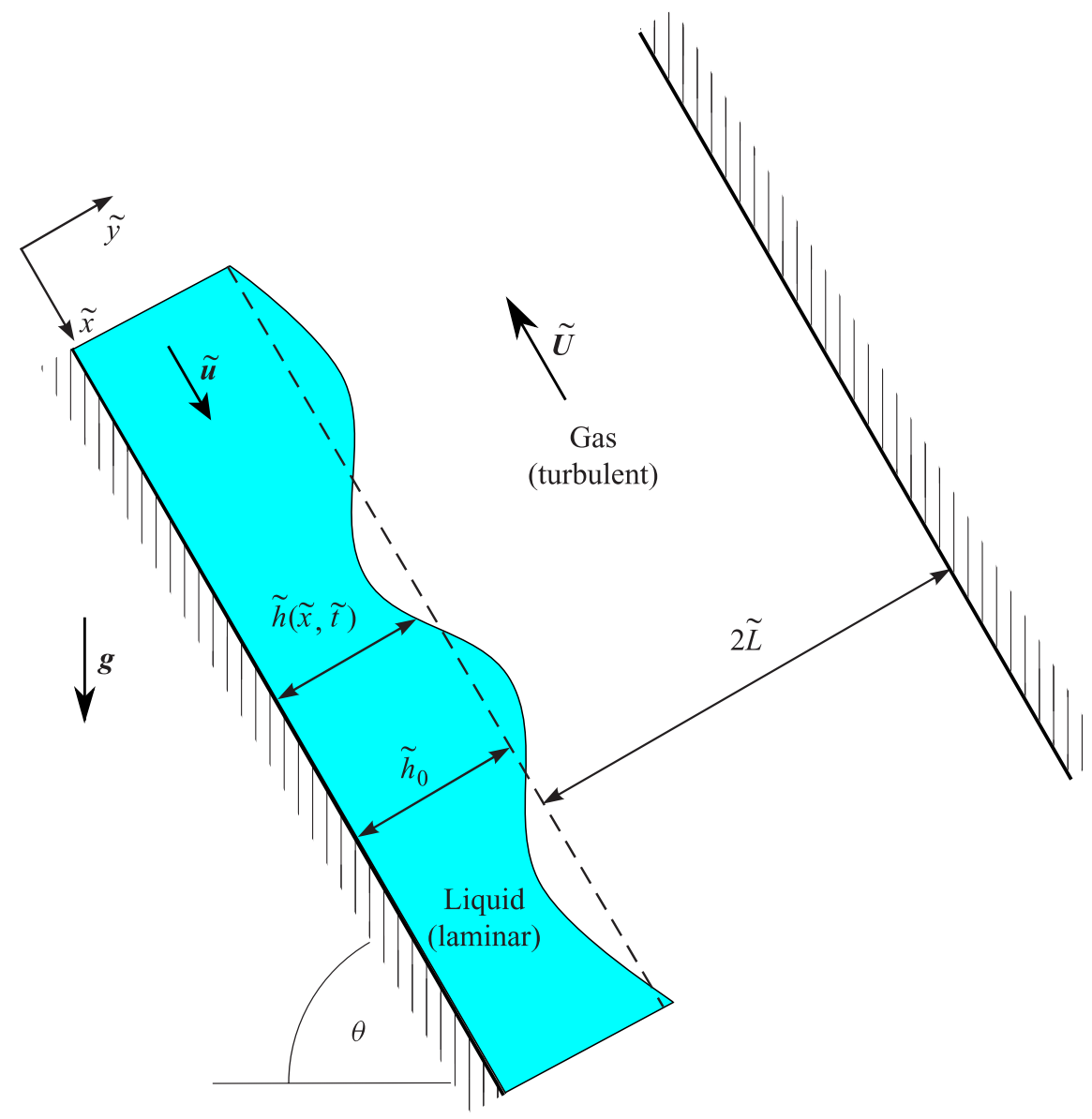

Figure 2. Schematic illustration of a falling liquid film in contact with a counter-current turbulent gas. $\theta$ is the inclination angle of the channel with respect to the horizontal.

where $\tilde{t}$ is time, $\tilde{\boldsymbol{u}}=(\tilde{u}, \tilde{v})$ is the liquid velocity, $\tilde{p}$ is the pressure and $\boldsymbol{g}=(g \sin \theta,-g \cos \theta)$ with $g$ denoting the acceleration due to gravity. The no-slip and no-penetration conditions at the bottom wall imply

$$
\tilde{\boldsymbol{u}}=0 \quad \text { at } \quad \tilde{y}=0 .
$$

The kinematic condition at the gas-liquid interface $\tilde{h}$ is

$$
\tilde{h}_{\tilde{t}}+\tilde{u} \tilde{h}_{\tilde{x}}=\tilde{v} \quad \text { at } \quad \tilde{y}=\tilde{h}(\tilde{x}, \tilde{t}) .
$$

The tangential stress balance is given by

$$
-\frac{\mu_{\ell}}{1+\tilde{h}_{\tilde{x}}^{2}}\left[2\left(\tilde{u}_{\tilde{x}}-\tilde{v}_{\tilde{y}}\right)-\left(\tilde{u}_{\tilde{y}}+\tilde{v}_{\tilde{x}}\right)\left(1-\tilde{h}_{\tilde{x}}^{2}\right)\right]=\tilde{\tau}_{w}[\tilde{h}] \quad \text { at } \quad \tilde{y}=\tilde{h}(\tilde{x}, \tilde{t}),
$$

where $\tilde{\tau}_{w}[\tilde{h}]$ is the tangential stress at the interface due to the turbulent gas flow. The normal stress balance can be written as

$$
\tilde{p}=\tilde{P}_{w}[\tilde{h}]+\frac{2 \mu_{\ell}}{1+\tilde{h}_{\tilde{x}}^{2}}\left[\tilde{u}_{\tilde{x}} \tilde{h}_{\tilde{x}}^{2}-\left(\tilde{u}_{\tilde{y}}+\tilde{v}_{\tilde{x}}\right) \tilde{h}_{\tilde{x}}+\tilde{v}_{\tilde{y}}\right]-\frac{\sigma \tilde{h}_{\tilde{x} \tilde{x}}}{\left(1+\tilde{h}_{\tilde{x}}^{2}\right)^{3 / 2}} \quad \text { at } \quad \tilde{y}=\tilde{h}(\tilde{x}, \tilde{t}),
$$

where $\tilde{P}_{w}[\tilde{h}]$ is the pressure exerted at the interface by the turbulent gas. Expressions for 
the tangential and normal stresses exerted by the turbulent gas on the liquid film have been obtained by Tseluiko \& Kalliadasis (2011) and turn out to be non-local operators of the interface shape. These expressions will be given below in dimensionless forms. The basic hypotheses by Tseluiko \& Kalliadasis (2011) are that the dynamic viscosity of the gas is much smaller than that of the liquid, that the typical velocity in the liquid and that of the interfacial waves is much smaller than the typical velocity of the gas and that of a 'linear response' i.e. the interface, written as a superposition of Fourier harmonics of wavenumber say $\alpha_{n}$ (assuming periodicity) induces tangential and normal stresses which are also superposition of Fourier harmonics of the same wavenumber $\alpha_{n}$. In choosing the turbulence model, the motivation of Tseluiko \& Kalliadasis (2011) was to utilise a simple model that gives good agreement with experiments and allows for an analytical progress to be made with the problem. Therefore, Tseluiko \& Kalliadasis (2011) used Prandtl's mixing length theory with the mixing length modelled by the van Driest equation. The drawbacks of the standard mixing length model are well known (e.g. it predicts that the apparent viscosity should vanish in the middle of the channel, which is actually not the case), however it still provides good agreement with experiments in many situations (e.g. Schlichting 2000). To model turbulent flow over a wavy surface, Tseluiko \& Kalliadasis (2011) chose to work in orthogonal boundary-layer coordinates introduced by Benjamin (1959), in which the wall to leading order coincides with a coordinate line and far from the wall the coordinate lines of the curvilinear system approach exponentially those of the Cartesian system. Tseluiko \& Kalliadasis (2011) assumed that the waviness-induced turbulent Reynolds stresses vanish in such coordinates (which implicitly provides a model for the waviness-induced Reynolds stresses in Cartesian coordinates) and demonstrated by comparing with experimental results presented in Thorsness et al. (1978) that such modelling is capable of capturing satisfactorily the effects of turbulent gas flow over a wavy surface (see figures 5-11 in Tseluiko \& Kalliadasis 2011).

To make the above equations dimensionless, we choose the following scales for length $\left(l_{c}\right)$, velocity $\left(\tilde{u}_{c}\right)$, time $\left(\tilde{t}_{c}\right)$ and pressure $\left(\tilde{p}_{c}\right)$ :

$$
\tilde{l}_{c}=\tilde{h}_{0} ; \quad \tilde{u}_{c}=\frac{\rho_{\ell} g \tilde{h}_{0}^{2} \sin \theta}{2 \mu_{\ell}} ; \quad \tilde{t}_{c}=\frac{\tilde{h}_{0}}{\tilde{u}_{c}} ; \quad \tilde{p}_{c}=\frac{\mu_{\ell} \tilde{u}_{c}}{\tilde{h}_{0}} .
$$

Using these, the Navier-Stokes equations are written in dimensionless form as follows

$$
\begin{aligned}
\operatorname{Re}\left(u_{t}+u u_{x}+v u_{y}\right) & =-p_{x}+u_{x x}+u_{y y}+2, \\
\operatorname{Re}\left(v_{t}+u v_{x}+v v_{y}\right) & =-p_{y}+v_{x x}+v_{y y}-2 \cot \theta, \\
u_{x}+v_{y} & =0,
\end{aligned}
$$

where

$$
R e=\frac{\rho_{\ell}^{2} g \tilde{h}_{0}^{3} \sin \theta}{2 \mu_{\ell}^{2}}
$$

is the Reynolds number. At the bottom wall, we have

$$
u=v=0, \quad \text { at } \quad y=0 .
$$

On the interface, the dimensionless kinematic condition is given by

$$
h_{t}+u h_{x}=v, \quad \text { at } \quad y=h(x, t),
$$

with the dimensionless tangential stress condition given by

$$
-\frac{1}{1+h_{x}^{2}}\left[2\left(u_{x}-v_{y}\right) h_{x}-\left(u_{y}+v_{x}\right)\left(1-h_{x}^{2}\right)\right]=\hat{\tau}_{w}[h] \quad \text { at } \quad y=h(x, t),
$$


where

$$
\hat{\tau}_{w}[h]=\hat{\tau}_{w 0}+\hat{\tau}_{w 0} \nu \hat{\tau}_{w 1}[h]
$$

with

$$
\hat{\tau}_{w 0}=\frac{\tilde{h}_{0} T_{w} \tau_{w 0}}{\mu_{l} \tilde{u}_{0}}, \quad \nu=\frac{\tilde{h}_{0} \rho_{g} U_{f}^{*}}{\mu_{g}}, \quad \hat{\tau}_{w 1}[h] \equiv \sum_{\substack{n=-\infty \\ n \neq 0}}^{\infty} h_{n} \tau_{w 1}\left(\alpha_{n} / \nu\right) \mathrm{e}^{\mathrm{i} \alpha_{n} x},
$$

where $U_{f}^{*}$ is the frictional velocity in the turbulent gas flow, and $\alpha_{n}$ is the wavenumber. The dimensionless form of the normal stress balance condition can be written as

$$
p=\hat{P}_{w}[h]+\frac{2}{1+h_{x}^{2}}\left[u_{x} h_{x}^{2}-\left(u_{y}+v_{x}\right) h_{x}+v_{y}\right]-\frac{R e W e h_{x x}}{\left(1+h_{x}^{2}\right)^{3 / 2}}, \quad \text { at } \quad y=h(x, t),
$$

where

$$
\begin{gathered}
\widehat{P}_{w}[h]=\widehat{P}_{0}+\left|\hat{\tau}_{w 0}\right| \nu \widehat{P}_{1}[h] \\
\widehat{P}_{0}=\left|\hat{\tau}_{w 0}\right| P_{0}=\widehat{P}_{0}^{a}+\widehat{P}_{0}^{b} x, \quad \widehat{P}_{1}[h] \equiv \sum_{\substack{n=-\infty \\
n \neq 0}}^{\infty} h_{n} P_{w 1}\left(\alpha_{n} / \nu\right) \mathrm{e}^{\mathrm{i} \alpha_{n} x}, \\
\widehat{P}_{0}^{a}=\left|\hat{\tau}_{w 0}\right| P_{0}{ }^{a}, \quad \widehat{P}_{0}^{b}=-\frac{\hat{\tau}_{w 0}}{\hat{L}}
\end{gathered}
$$

with $P_{0}{ }^{a}$ being the reference pressure and $\hat{L}$ the dimensionless width of the channel. We note that functions $\tau_{w 1}$ and $P_{w 1}$ are defined by

$$
\tau_{w 1}(\alpha)=\psi_{1}^{\prime \prime}(0)-2 \alpha, \quad P_{w 1}(\alpha)=-\mathrm{i}\left[\frac{1}{L}+2 \alpha-\psi_{1}^{\prime \prime \prime}(0)\right],
$$

where $L=\rho_{g} U_{f}^{*} \tilde{L} / \mu_{g}$, with $\tilde{L}$ denoting the half distance between the undisturbed film surface and the upper wall, and $\psi_{1}(y)$ is an interface-waviness-induced perturbation to the gas stream function that is obtained for each interface wavenumber $\alpha$ by numerically solving a two-point boundary-value problem. We omit the details here, which can be found in Tseluiko \& Kalliadasis (2011). The dimensionless parameter $W e$ is the Weber number that signifies the ratio of surface tension forces to the inertial forces, given by

$$
W e=\frac{4 K a}{R e_{0}^{5 / 3} \sin ^{2} \theta}
$$

with $R e=\left(R e_{0} \sin \theta\right) / 2$, and $K a=\sigma \rho_{\ell}^{1 / 3} /\left(g^{1 / 3} \mu_{\ell}^{4 / 3}\right)$ is the Kapitza number that depends on the properties of the liquid only. For convenience we can write

$$
\hat{\tau}_{w 0}=\frac{2}{R e_{0}^{1 / 3} \sin \theta} \Theta \tau_{w 0}, \quad \nu=N R e_{0}^{1 / 3} \Theta^{1 / 2},
$$

where

$$
\Theta=\frac{\left|T_{w}\right|}{\rho_{l}^{1 / 3} \mathrm{~g}^{2 / 3} \mu_{l}^{2 / 3}}, \quad N=\frac{\mu_{l} \rho_{g}^{1 / 2}}{\mu_{g} \rho_{l}^{1 / 2}} .
$$

The parameter $\Theta$ controls the gas shear-stress strength, whereas the parameter $N$ depends only on the densities and viscosities of the liquid and the gas. 


\section{Low-dimensional models}

Solving the full Navier-Stokes equations for the gas and liquid problems is an arduous task. To alleviate this difficulty, low-dimensional models that capture the essential physics of the full problem for a range of values in the parameter space were developed by Tseluiko \& Kalliadasis (2011). Here, we recall only the main ideas and the final equations, for details on the derivation the reader is referred to Tseluiko \& Kalliadasis (2011).

\subsection{Long-wave model}

To derive a long-wave model, it is assumed that interfacial waves have a long wavelength when compared to the thickness of the film. Applying the classical long-wave expansion of the velocity and pressure fields, first proposed by Benney (1966), Tseluiko \& Kalliadasis (2011) obtain

$$
\begin{aligned}
h_{t}+2 h^{2} h_{x}+\left(\left(\frac{8}{15} R e h^{6}-\frac{2}{3} \cot \theta h^{3}\right) h_{x}\right. & \left.+\frac{1}{3} R e W e h^{3} h_{x x x}\right)_{x} \\
& +\frac{\hat{\tau}_{w 0}}{2}\left(h^{2}\left(1+\frac{8 R e}{15} h^{3} h_{x}+\nu \hat{\tau}_{w 1}[h]\right)\right)_{x}=0 .
\end{aligned}
$$

By ignoring the terms multiplying $\hat{\tau}_{w 0}$ in the parentheses that represent the effect of turbulent gas flow, we recover the classic Benney's equation for the case of a falling film. Although this nonlinear partial differential equation is probably the simplest possible model that correctly predicts the linear stability threshold for long waves, its disadvantage is that it suffers finite-time blow up when $R e$ exceeds a limiting value.

\subsection{Weighted integral boundary layer (WIBL) model}

The shortcomings of the long-wave model can be overcome with the methodology developed by Ruyer-Quil \& Manneville (1998, 2000) to model falling films on inclined planes in the region of small-to-moderate Reynolds numbers. The starting point for this derivation is the first-order boundary layer equations. Combining these equations with a weighted-residuals technique where the velocity components are expanded in terms of polynomial test functions satisfying the boundary conditions yields the residual. Applying this methodology to our problem and using a simple Galerkin projection with just one test function yields a set of coupled equations for the evolution of film thickness $(h)$ and the local flow rate $(q)$ :

$$
\begin{aligned}
q_{t}=- & \frac{17}{7} \frac{q q_{x}}{h}+\frac{9}{7} \frac{q^{2} h_{x}}{h^{2}}-\frac{5}{2 R e} \frac{q}{h^{2}}+\frac{5}{3 R e} h-\frac{5 \cot \theta}{3 R e} h h_{x}+\frac{5 W e}{6} h h_{x x x} \\
+ & \hat{\tau}_{w 0}\left(\frac{5}{4 R e}-\frac{19 \hat{\tau}_{w 0}}{672} h^{2} h_{x}-\frac{19}{336} h q_{x}-\frac{5}{112} q h_{x}+\frac{5 \operatorname{sign}\left(\hat{\tau}_{w 0}\right) \nu}{4 R e} \hat{\tau}_{w 1}[h]\right), \\
h_{t}+q_{x} & =0 .
\end{aligned}
$$

The terms multiplying $\hat{\tau}_{w 0}$ correspond to the influence of the turbulent gas on the liquid film dynamics, and by setting $\hat{\tau}_{w 0}$ to zero we recover the case of a falling film (Ruyer-Quil \& Manneville 2000). This WIBL model predicts the correct value for the linear stability threshold, but is also applicable for moderately large values of $R e$. We note that although in many applications involving gas-liquid flows the liquid Reynolds number is large, there are applications where it can take moderate values, for example, in desiccant cooling systems (see e.g. Ali et al. 2003; Mouza et al. 2005) and in the cooling of microelectronic 
devices (see e.g. Kabov et al. 2007). In addition, there are several experiments that are preformed at moderate liquid Reynolds numbers (see e.g. Semyonov 1944; Clift et al. 1966; Zapke \& Kröger 2000b).

\section{Linear stability analysis}

\subsection{Formulation of the OS problem}

To obtain the base state solution, we shall assume the flow to be steady and unidirectional with the interface being flat. The governing equations simplify to

$$
\begin{gathered}
-\widehat{P}_{0 x}+u_{0 y y}+2=0, \\
\widehat{P}_{0 y}+2 \cot \theta=0 .
\end{gathered}
$$

The above equations are to be solved subject to the boundary conditions

$$
u_{0}(0)=0 \quad \text { and } \quad u_{0 y}(1)=\hat{\tau}_{w 0} .
$$

Integrating equation (4.1a) twice with respect to $y$ and applying the boundary conditions (4.2), we obtain

$$
u_{0}=\left(\frac{\widehat{P}_{0 x}}{2}-1\right)\left(y^{2}-2 y\right)+\hat{\tau}_{w 0} y .
$$

Further by making use of the relation (2.20) and taking the channel to be much wider than the liquid film thickness, equation (4.3) simplifies to

$$
u_{0}=-\left(y^{2}-2 y\right)+\hat{\tau}_{w 0} y .
$$

The base-state stream function can be obtained by integrating (4.4) with respect to $y$ :

$$
\psi_{0}=-\left(\frac{y^{3}}{3}-y^{2}\right)+\frac{\hat{\tau}_{w 0}}{2} y^{2} .
$$

We eliminate the pressure terms from the Navier-Stokes equations (2.8) and (2.9) by cross-differentiation, followed by making use of the definition of the stream function, $u=\Psi_{y}, v=-\Psi_{x}$ to obtain

$$
\left(\frac{\partial}{\partial t}+\frac{\partial \Psi}{\partial y} \frac{\partial}{\partial x}-\frac{\partial \Psi}{\partial x} \frac{\partial}{\partial y}\right) \nabla^{2} \Psi=\frac{1}{R e} \nabla^{4} \Psi
$$

As the next step, we introduce infinitesimal perturbations to the base flow by writing $\Psi=\psi_{0}+\epsilon \psi_{1}+O\left(\epsilon^{2}\right)$, where $\epsilon \ll 1$. Substituting this in (4.6) and retaining only the linear terms, yields

$$
\left(\frac{\partial}{\partial t}+u_{0} \frac{\partial}{\partial x}\right) \nabla^{2} \psi_{1}-u_{0}^{\prime \prime} \frac{\partial \psi_{1}}{\partial x}=\frac{1}{R e} \nabla^{4} \psi_{1}
$$

where prime denotes differentiation with respect to $y$, and $u_{0}$ is the base-state velocity given by $u_{0}=\psi_{0}^{\prime}$. Further, by writing the perturbation $\psi_{1}$ in the form of normal modes, $\psi_{1}=\phi(y) \mathrm{e}^{\mathrm{i} k(x-c t)},(4.7)$ results in the following classical OS equation:

$$
\phi^{\prime \prime \prime \prime}-2 k^{2} \phi^{\prime \prime}+k^{4} \phi=\mathrm{i} k R e\left[\left(u_{0}-c\right)\left(\phi^{\prime \prime}-k^{2} \phi\right)-u_{0}^{\prime \prime} \phi\right] .
$$

The no-penetration and no-slip conditions at the bottom wall, respectively, imply

$$
\phi(0)=0, \quad \phi^{\prime}(0)=0 .
$$


The perturbation to the flat interface can be represented as $y=h+\epsilon \eta(x, t)$. Therefore, the waveform of the interface disturbance is given by $\eta(x, t)=A_{1} \mathrm{e}^{\mathrm{i} k(x-c t)}$, where $A_{1}$ is a complex quantity. Substituting this in (2.13), we obtain the linearised form of the kinematic condition

$$
\phi(1)=\left(c-1-\hat{\tau}_{w 0}\right) A_{1}
$$

From the tangential stress balance condition (2.14), we have

$$
\phi^{\prime \prime}(1)+k^{2} \phi(1)-\left[\left|\hat{\tau}_{w 0}\right| \nu \tau_{w 1}(k / \nu)+2\right] A_{1}=0 .
$$

The normal stress balance condition (2.17) becomes

$$
\begin{aligned}
& \phi^{\prime \prime \prime}(1)+k\left[\mathrm{i} R e\left(c-1-\hat{\tau}_{w 0}\right)-3 k\right] \phi^{\prime}(1)+\mathrm{i} k \operatorname{Re} \hat{\tau}_{w 0} \phi(1) \\
& \quad-k\left[2 \mathrm{i} \cot \theta+\mathrm{i} k^{2} W e R e+2 k \hat{\tau}_{w 0}+\mathrm{i}\left|\hat{\tau}_{w 0}\right| \nu P_{w 1}(k / \nu)\right] A_{1}=0 .
\end{aligned}
$$

The OS equation (4.8) together with the boundary conditions (4.9), (4.10), (4.11) and (4.12) constitute a generalised eigenvalue problem, with $c$ the eigenvalue.

\subsection{Dispersion relations for low-dimensional models}

To understand the linear stability of the flat-film solution $(h=1)$ in a long-wave model, we linearise $(3.1)$ by substituting $h=1+f$, where $|f| \ll 1$, and retaining only the first-order terms, we obtain

$$
f_{t}+a f_{x}+b f_{x x}+c f_{x x x x}+d\left(\hat{\tau}_{w 1}[f]\right)_{x}=0
$$

where

$$
a=2+\hat{\tau}_{w 0}, \quad b=\frac{8 R e}{15}-\frac{2 \cot \theta}{3}+\frac{4 R e \hat{\tau}_{w 0}}{15}, \quad c=\frac{R e W e}{3}, \quad d=\frac{\left|\hat{\tau}_{w 0}\right| \nu}{2} .
$$

The linearised long-wave equation (4.13) represents the evolution of an infinitesimal perturbation $f$. We look for solutions in the form of normal modes, $f \propto \mathrm{e}^{s(k) t+\mathrm{i} k x}$, to obtain the following dispersion relation:

$$
s(k)=-\mathrm{i} a k+b k^{2}-c k^{4}-\mathrm{i} d k \tau_{w 1}(k / \nu) .
$$

In a similar fashion, by substituting $h=1+f$ and $q=2 / 3+\hat{\tau}_{w 0} / 2+g$, where $|f| \ll 1$ and $|g| \ll 1$ in (3.2) and (3.3), the linearised form of the WIBL model is obtained as

$$
\begin{gathered}
f_{t}+g_{x}=0, \\
g_{t}=-\left(\frac{34}{21}+\frac{61}{48} \hat{\tau}_{w 0}\right) g_{x}-\frac{5}{2 R e} g+\frac{5 W e}{6} f_{x x x}+\left(\frac{4}{7}+\frac{139}{168} \hat{\tau}_{w 0}+\frac{13}{48} \hat{\tau}_{w 0}^{2}-\frac{5 \cot \theta}{3 R e}\right) f_{x} \\
+\frac{5}{2 R e}\left(2+\hat{\tau}_{w 0}\right) f+\frac{5 \operatorname{sign}\left(\hat{\tau}_{w 0}\right) \nu}{4 R e} \hat{\tau}_{w 0} \hat{\tau}_{w 1}[f] .
\end{gathered}
$$

As with the long-wave model, we look for solutions in the form of normal modes, $(f, g) \propto$ $\mathrm{e}^{s(k) t+\mathrm{i} k x}$ to obtain the following quadratic dispersion relation:

$$
\begin{aligned}
s^{2}(k)+ & \frac{1}{336 R e}\left[840+\mathrm{i} k \operatorname{Re}\left(427 \hat{\tau}_{w 0}+544\right)\right] s(k)+\frac{5 W e}{6} k^{4} \\
+ & \frac{1}{336 R e}\left[-\operatorname{Re}\left(192+278 \hat{\tau}_{w 0}+91 \hat{\tau}_{w 0}^{2}\right)+560 \cot \theta\right] k^{2} \\
& +\mathrm{i} \frac{5}{4 R e}\left[4+2 \hat{\tau}_{w 0}+\operatorname{sign}\left(\hat{\tau}_{w 0}\right) \hat{\tau}_{w 0} \nu \tau_{w 1}(k / \nu)\right] k=0 .
\end{aligned}
$$


The above set of dispersion relations (4.15), (4.18), along with the OS system, (4.8)(4.12) will be utilised in the stability calculations that will be presented in the following sections.

\section{Numerical results}

In this section we will discuss numerical results. We will mainly adopt helium (gas) and methanol (liquid) as working fluids, which were used, for example in the experiments of Zapke \& Kröger $(2000 a, b)$ on flooding in rectangular channels with an emphasis on geometrical effects. The physical properties of those are as follows:

$$
\begin{gathered}
\rho_{g}=0.165 \mathrm{~kg} / \mathrm{m}^{3}, \quad \mu_{g}=2 \times 10^{-5} \mathrm{~Pa} \mathrm{~s} \\
\rho_{\ell}=791 \mathrm{~kg} / \mathrm{m}^{3}, \quad \mu_{\ell}=5.75 \times 10^{-4} \mathrm{~Pa} \mathrm{~s}, \quad \sigma=22 \times 10^{-3} \mathrm{~N} / \mathrm{m},
\end{gathered}
$$

which in terms of dimensionless numbers in our formulation are given by

$$
K a=1988.5, \quad N=0.4 .
$$

\subsection{Temporal stability}

In order to analyse the temporal stability of the system, the wavenumber $k$ is taken to be real whereas $c$ is complex. This implies that the real part of $c$ gives the phase speed, whereas $k c_{i}$ is the growth rate of the perturbation with $c_{i}$ being the imaginary part of the complex quantity $c$.

\subsubsection{Case I: No gas flow}

To verify our numerical procedure, we first switch off the gas flow and examine if we recover the linear stability results for a falling film (see e.g. Yih 1963). This is realised by setting $\hat{\tau}_{w 0}=0, \tau_{w 1}(k / \nu)=0$ and $P_{w 1}(k / \nu)=0$. The asymptotic solution for small $k$ is given by

$$
\begin{gathered}
\phi=(z+1)^{2}+k\left[\frac{1}{240} \mathrm{i} R e z^{5}-\frac{1}{48} \mathrm{i} R e z^{4}+\frac{1}{6}\left(-\frac{3}{4} \mathrm{i} R e+\mathrm{i} \cot \theta\right) z^{3}\right. \\
\left.+\frac{1}{2}\left(-\frac{41}{120} \mathrm{i} R e+\frac{2}{3} \mathrm{i} \cot \theta+2\right) z^{2}+\left(-\frac{17}{240} \mathrm{i} R e+\frac{1}{6} \mathrm{i} \cot \theta+2\right) z+1\right]+\cdots \\
c=2+\mathrm{i} k\left(\frac{8}{15} R e-\frac{2}{3} \cot \theta\right)+\cdots
\end{gathered}
$$

whilst the growth rate is

$$
s_{r}=-\operatorname{Re}(\mathrm{i} k c) \equiv k^{2}\left(\frac{8}{15} R e-\frac{2}{3} \cot \theta\right) .
$$

The flat-film solution becomes unstable when $s_{r}>0$, from which one can deduce the classical result that the critical Reynolds number at which the instability sets in is

$$
R e_{c}=\frac{5}{4} \cot \theta
$$

Figure 3 shows the growth rate of the disturbance, $s_{r}$, as a function of Reynolds number, $R e$, with the wavenumber $k=0.001$ when $\theta=\pi / 4$ and $K a=1988.5$. Our computations by two different techniques, namely the 'FFT-based' and 'matrix-based', 


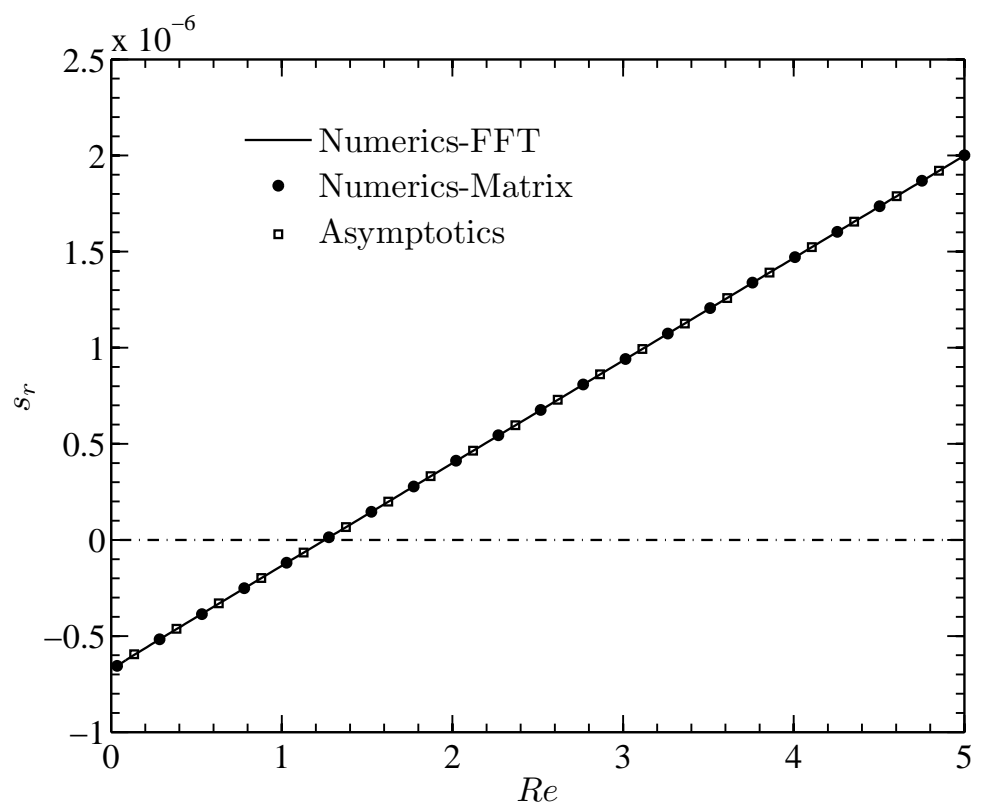

Figure 3. The growth rate, $s_{r}$, vs the Reynolds number, Re, for a falling liquid film when $\Theta=0, \theta=\pi / 4, k=0.001$ and $K a=1988.5$.

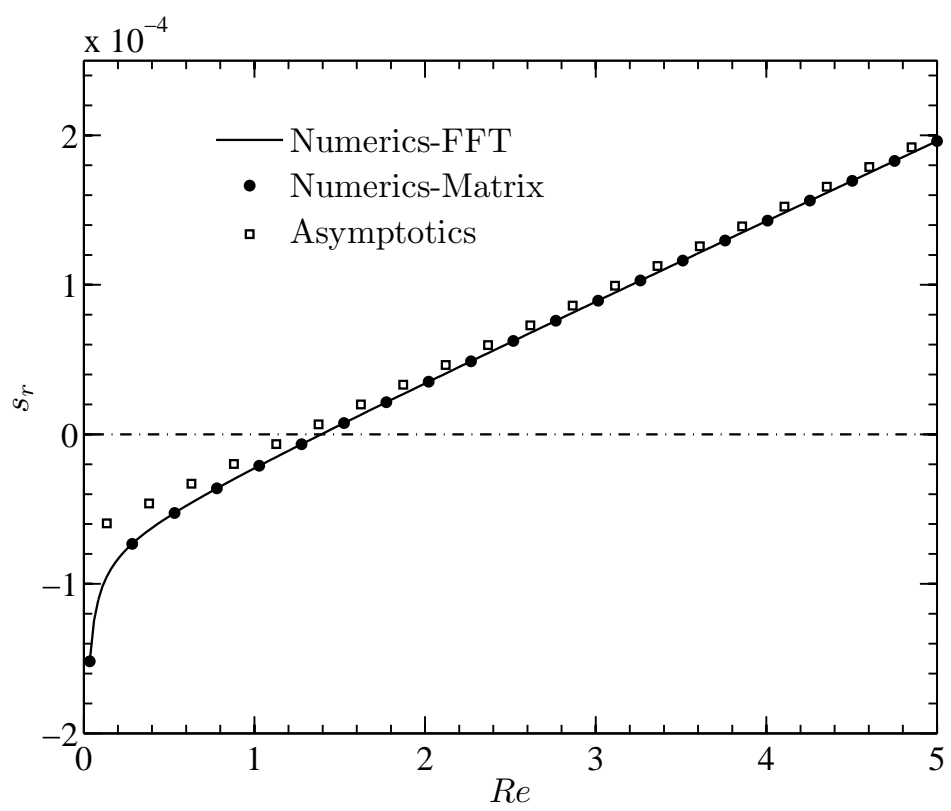

Figure 4. The growth rate, $s_{r}$, vs the Reynolds number, Re, for a falling liquid film when $\Theta=0, \theta=\pi / 4, k=0.01$ and $K a=1988.5$.

agree very well with the asymptotic result. For details on the numerical scheme, the reader is referred to Appendix A. Beyond the critical Reynolds number $\left(R e_{c} \equiv 5 \cot \theta / 4\right)$, the flat film solution becomes unstable and the growth rate increases with increasing $R e$. As we increase the wavenumber $(k=0.01)$ further, the growth rate increases for any given $R e$, but the numerics still predicts the critical Reynolds number very well (as shown in figure 4). 


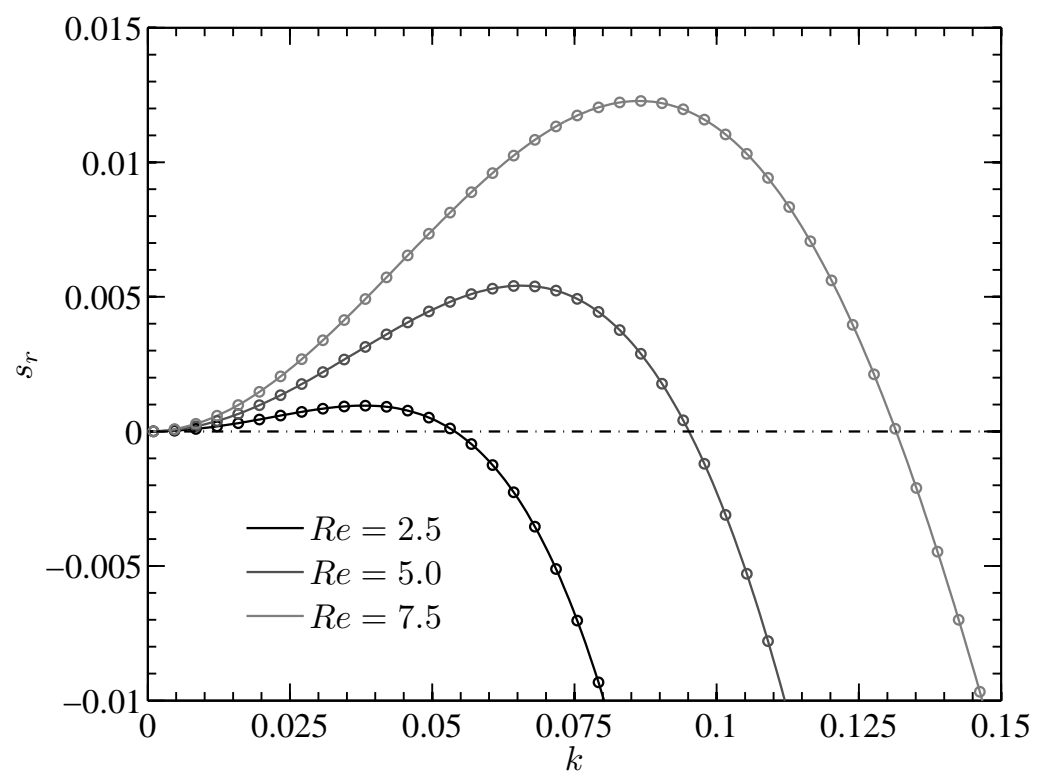

FiguRE 5. The growth rate, $s_{r}$, vs the wavenumber, $k$, for a falling liquid film when $\Theta=0$, $\theta=\pi / 2$ and $K a=1988.5$. The solid curves correspond to the solutions from the 'FFT-based' method and the open circles to the solutions from the 'Matrix-based' technique.

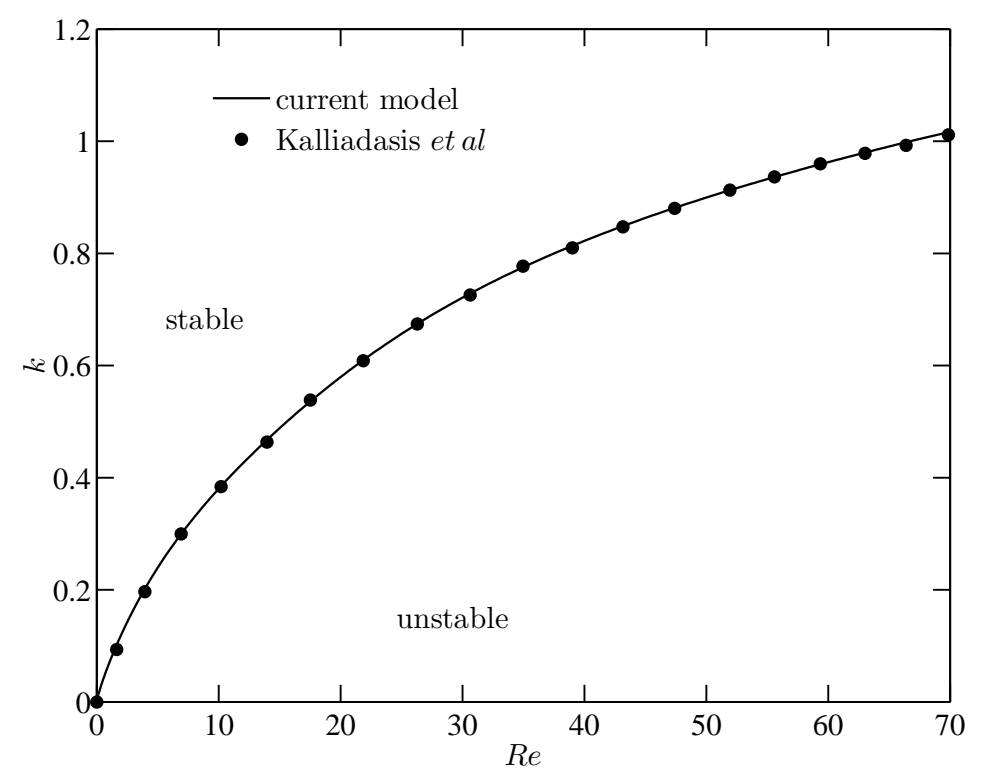

Figure 6 . The neutral stability curve for a vertically falling film $(\theta=\pi / 2)$ when $\Theta=0$ and $K a=528.8$. The solid curve corresponds to our OS system, whereas the closed circles correspond to the results of Kalliadasis et al. (2012).

Figure 5 shows the growth rates, corresponding to the dominant normal mode for the case of a vertically falling liquid film $(\theta=\pi / 2)$, for various $R e$. As can be observed from the plot, the vertically falling liquid film is unstable to long-wavelength disturbances (for any $R e$ ), and the band of unstable wavenumbers increases as $R e$ is increased. As the wavenumber is increased beyond a particular value, the growth rate becomes negative, implying that the short wave disturbances are damped. 


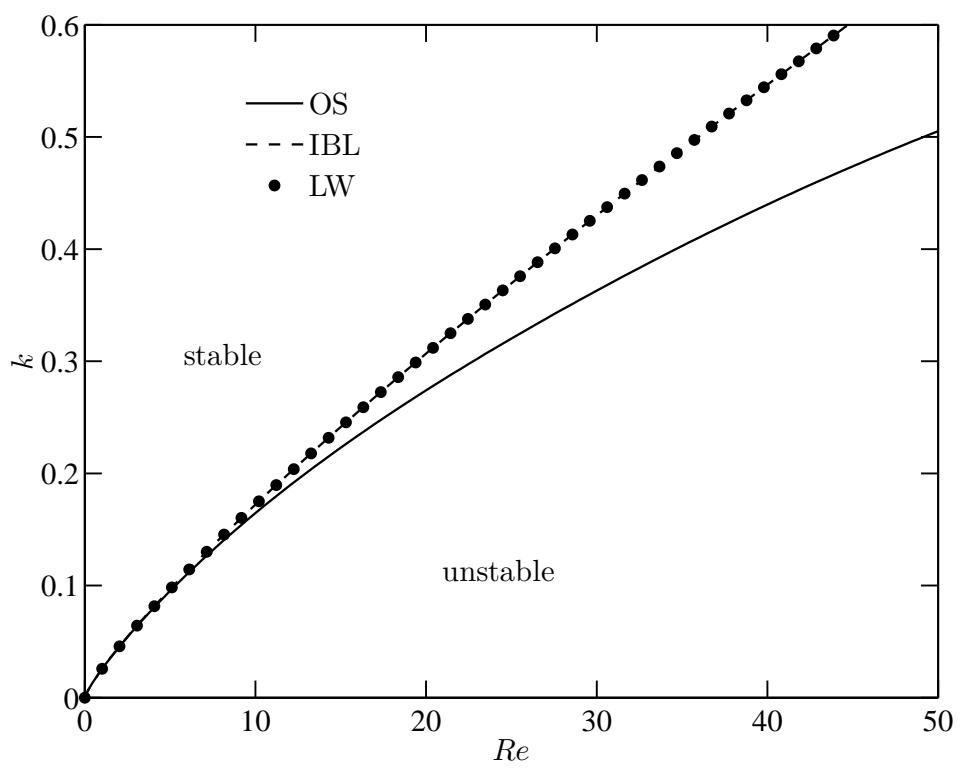

FIGURE 7 . The neutral stability curve for a vertically falling film $(\theta=\pi / 2)$ when $\Theta=0$ and $K a=1988.5$. The solid curve corresponds to our OS system, the closed circles correspond to the long-wave model, and the dashed curve corresponds to the WIBL model.

Another way to assess the linear stability of the falling film system is to consider the neutral stability curves. In figure 6, we compare the neutral curves computed from our OS formulation (with the gas flow switched-off) with those of Kalliadasis et al. (2012) for the parameter values, $K a=528.8$ and $\theta=\pi / 2$, and we find excellent agreement.

Finally, we compare the neutral curves obtained by solving the dispersion relations of the long-wave model (4.15) and the WIBL model (4.18) with the OS system for the vertical configuration $(\theta=\pi / 2)$ and $K a=1988.5$. From figure 7 , it can be observed that the long-wave and WIBL models correctly predict the linear stability threshold for $R e$ up to $\simeq 10$, beyond which the agreement becomes poorer as the inertial effects become important that are not captured by the low-dimensional models. It can also be observed that the results for the WIBL model are in much better agreement with the OS results than those of long-wave model.

\subsubsection{Case II: with gas flow}

Following a few reasonable approximations in the gas flow (Lin et al. 2013), the asymptotic solution for the falling film in the presence of counter-current gas is given by

$$
\begin{gathered}
\phi=(z+1)^{2}+k\left[\frac{1}{480} \mathrm{i} \operatorname{Re}\left(2+\hat{\tau}_{w 0}\right) z^{5}-\frac{1}{96} \mathrm{i} \operatorname{Re}\left(2+\hat{\tau}_{w 0}\right) z^{4}-\frac{1}{48} \mathrm{i}\left(3 \operatorname{Re}\left(2+\hat{\tau}_{w 0}\right)\right.\right. \\
+\cot \theta) z^{3}+\frac{1}{480}\left(-\mathrm{i} \operatorname{Re}\left(2+\hat{\tau}_{w 0}\right)+160 \mathrm{i} \cot \theta+480\right) z^{2} \\
\left.+\frac{1}{480}\left(-17 \mathrm{i} \operatorname{Re}\left(2+\hat{\tau}_{w 0}\right)+80 \mathrm{i} \cot \theta+960\right) z+1\right]+\cdots \\
c=2+\hat{\tau}_{w 0}-2 k\left|\hat{\tau}_{w 0}\right|+\mathrm{i} k\left(\frac{4 R e}{15}\left(2+\hat{\tau}_{w 0}\right)-\frac{2}{3} \cot \theta\right)+\cdots .
\end{gathered}
$$



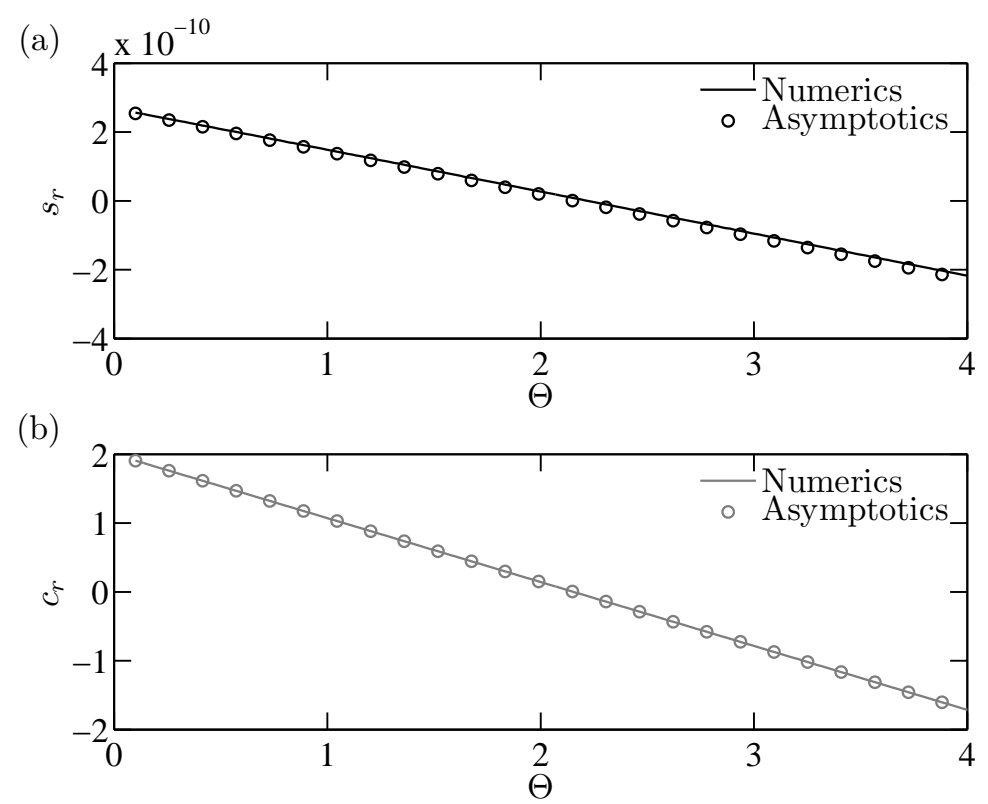

FiguRE 8. (a) The growth rate, $s_{r}$, vs the gas parameter, $\Theta$, and (b) the phase speed, $c_{r}$, vs the gas parameter, $\Theta$, for a falling liquid film in the presence of counter-current gas when $\theta=\pi / 2$, $k=10^{-5}, \mathrm{~N}=0.4$ and $K a=1988.5$

Therefore, the growth rate is given by

$$
s_{r}=k^{2}\left(\frac{4 R e}{15}\left(2+\hat{\tau}_{w 0}\right)-\frac{2}{3} \cot \theta\right) .
$$

The details of the solution procedure is provided in Appendix B. A comparison of the growth rates and phase speed of the disturbance obtained from asymptotic and numerical approaches is displayed in figure 8, which shows a good agreement. However, the agreement deteriorates when the wavenumber of the disturbance waves is taken to be sufficiently large. Therefore, we resort to numerical treatment of the OS equations and the low-dimensional models.

First, we present the neutral curves of the long-wave model (figure 9), the WIBL model (figure 10) and the OS problem (figure 11). We can observe that the stability boundaries obtained from the long-wave and WIBL models are slightly different from the OS results, especially for larger values of $\Theta$. It is also evident that the results for the WIBL model are in better agreement with the OS results than the results for the long-wave model, at least for $\Theta$ up to the value 2.5. We can observe that for the long-wave model, the region of stability increases with increasing the gas shear for larger values of $R e$. The behaviour is similar for the WIBL model but starts to change for larger values of $\Theta$. The OS results also show similar behaviour for smaller values of $\Theta$, but this behaviour drastically changes for larger values of $\Theta$. This qualitative difference between the results for the reduced model equations and the OS results is attributed to the fact that a consistent derivation of the reduced models (see Tseluiko \& Kalliadasis 2011) is done under the assumptions which imply that the normal stress imposed by the turbulent gas is of higher order and is therefore neglected, whereas for the considered helium-methanol system and for sufficiently large values of the gas parameter $\Theta$, it turns out that the energy production due to the normal stress imposed by the turbulent gas dominates over 


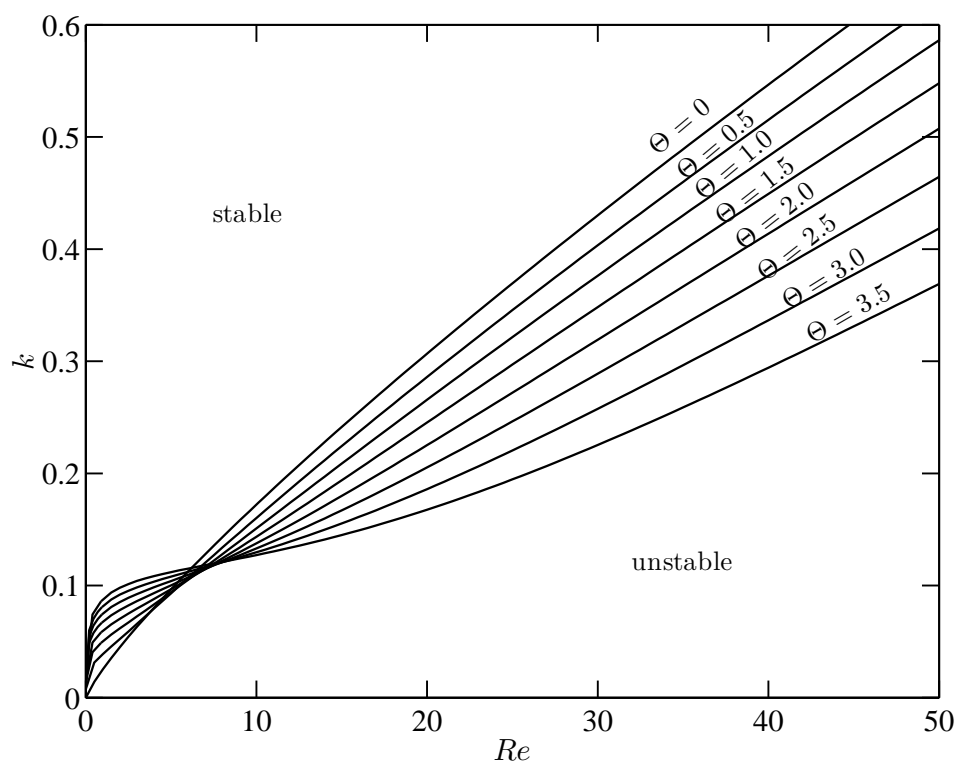

FIGURE 9. The neutral stability curves from the long-wave model for a vertically falling film in contact with counter-current gas flow for various values of gas shear $\Theta$, when $\theta=\pi / 2$, $K a=1988.5$ and $N=0.4$.

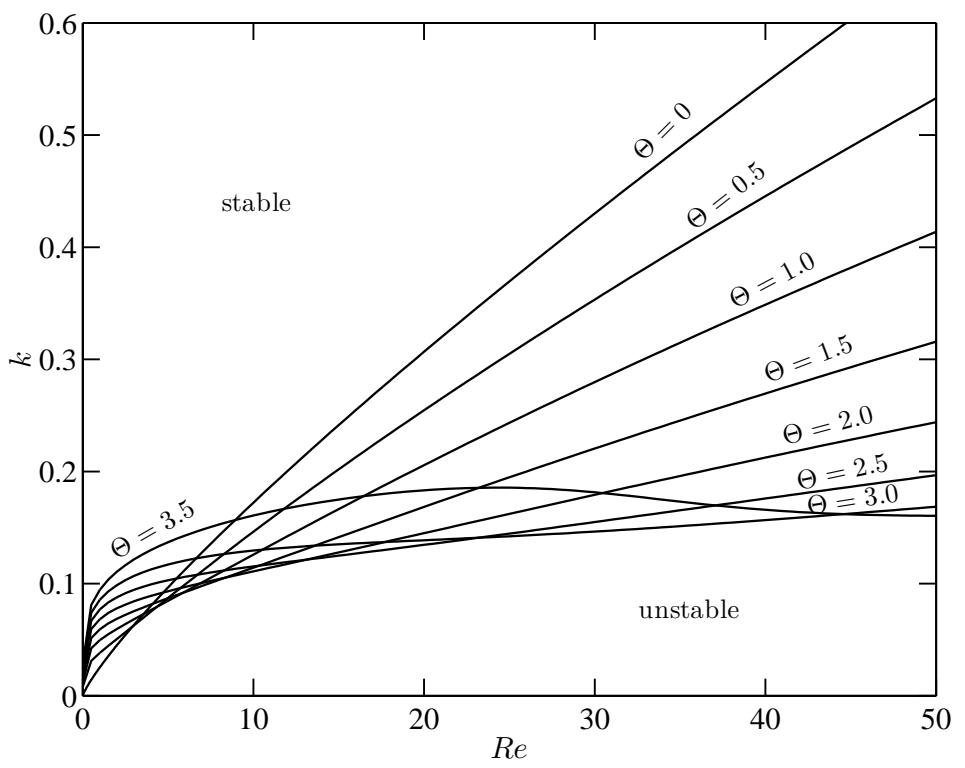

FIgURE 10. The neutral stability curves from the WIBL model for a vertically falling film in contact with counter-current gas flow for various values of gas shear $\Theta$, when $\theta=\pi / 2$, $K a=1988.5$ and $N=0.4$.

the energy production due to the tangential stress imposed by the turbulent gas (as is evident from $\S 5.2$ ).

To achieve a better comparison between the various models, we plot the stability boundary in the $k-\Theta$ plane for two different values of the Reynolds number, $R e=5$ and 18. It can be seen from figure 12 that both the long-wave and the WIBL model show 


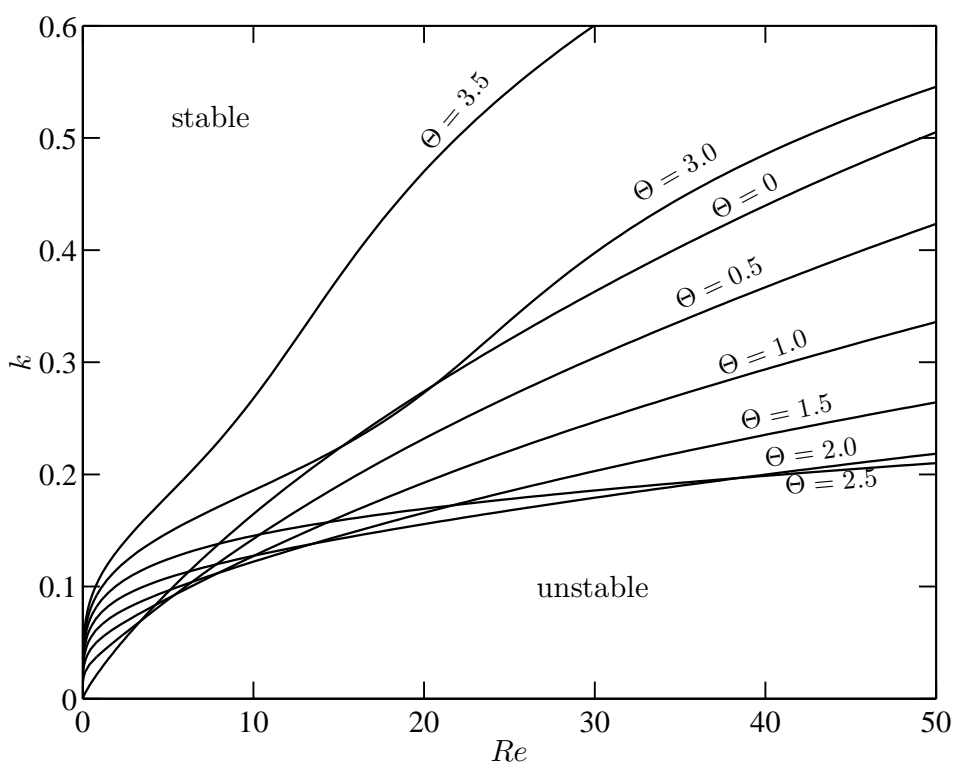

FIGURE 11. The neutral stability curves from the OS equations for a vertically falling film in contact with counter-current gas flow for various values of gas shear $\Theta$, when $\theta=\pi / 2$, $K a=1988.5$ and $N=0.4$.

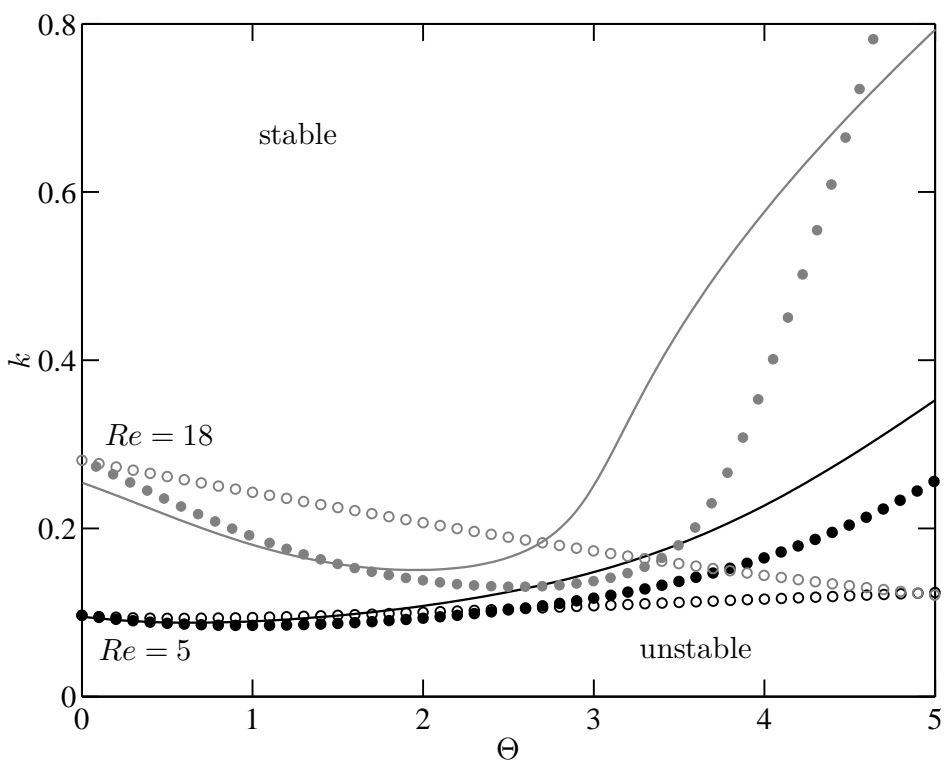

FIGURE 12. The neutral stability curves from the different formulations: the solid curves correspond to the OS equation, the open circles to the long-wave model and the closed circles to the WIBL model, when $\theta=\pi / 2, K a=1988.5$ and $N=0.4$. The black and grey shadings correspond to $R e=5$ and $R e=18$, respectively.

good agreement with the OS results for $R e=5$, but as the Reynolds number is increased to $R e=18$, the WIBL model overall shows better agreement with the OS results than the long-wave model. 


\subsection{Energy analysis}

To investigate the mechanism of instability in the gas-liquid system, we adopt a mechanical energy balance formulation (Smith \& Davis 1982; Hooper \& Boyd 1983; Kelly et al. 1989). The energy balance equation is derived by multiplying the linear equation for the perturbation evolution with $\left(u_{1}, v_{1}\right)$, further by integrating across the interface thickness and over a wavelength $\left(L_{x}=2 \pi / k_{m}\right)$, where $k_{m}$ is the wavenumber corresponding to the maximum growth rate. The main ideas in the derivation are given below. The linearised equations and the boundary conditions governing the evolution of perturbations are given by

$$
\begin{gathered}
\operatorname{Re}\left(u_{1 t}+u_{0} u_{1 x}+v_{1} u_{0 y}\right)=-p_{1 x}+u_{1 x x}+u_{1 y y}, \\
\operatorname{Re}\left(v_{1 t}+u_{0} v_{1 x}\right)=-p_{1 y}+v_{1 x x}+v_{1 y y}, \\
u_{1 x}+v_{1 y}=0, \\
u_{1}=v_{1}=0, \quad \text { at } y=0, \\
\eta_{t}+\left(1+\hat{\tau}_{w 0}\right) \eta_{x}=v_{1}, \quad \text { at } y=1, \\
u_{1 y}+v_{1 x}=\left[\left|\hat{\tau}_{w 0}\right| \nu \tau_{w 1}(k / \nu)+2\right] \eta, \quad \text { at } y=1,
\end{gathered}
$$

and

$$
p_{1}=\left[\left|\hat{\tau}_{w 0}\right| \nu P_{w 1}(k / \nu)+2 \cot \theta\right] \eta-R e W e \eta_{x x}-2 \hat{\tau}_{w 0} \eta_{x}-2 u_{1 x}, \quad \text { at } \quad y=1,
$$

where $\eta$ corresponds to the deflection in the free surface about $h=1$. Multiplying (5.8) by $u_{1}$ and (5.9) by $v_{1}$, adding the results and integrating over the film thickness and the wavelength $\left(L_{x}\right)$ we obtain

$$
\begin{aligned}
\frac{R e}{2 L_{x}} \frac{d}{d t} \int_{0}^{1} \int_{0}^{L_{x}} & \left(u_{1}^{2}+v_{1}^{2}\right) \mathrm{d} x \mathrm{~d} y=-\frac{R e}{L_{x}} \int_{0}^{1} \int_{0}^{L_{x}} u_{0 y} u_{1} v_{1} \mathrm{~d} x \mathrm{~d} y \\
- & \frac{1}{L_{x}} \int_{0}^{1} \int_{0}^{L_{x}} u_{1} p_{1 x} \mathrm{~d} x \mathrm{~d} y-\frac{1}{L_{x}} \int_{0}^{1} \int_{0}^{L_{x}} v_{1} p_{1 y} \mathrm{~d} x \mathrm{~d} y \\
& +\frac{1}{L_{x}} \int_{0}^{1} \int_{0}^{L_{x}}\left(u_{1} u_{1 x x}+u_{1} u_{1 y y}+v_{1} v_{1 x x}+v_{1} v_{1 y y}\right) \mathrm{d} x \mathrm{~d} y
\end{aligned}
$$

On integrating (5.15) by parts, followed by substituting $p_{1}$ from (5.14) results in

$$
\begin{aligned}
& \frac{R e}{2 L_{x}} \frac{d}{d t} \int_{0}^{1} \int_{0}^{L_{x}}\left(u_{1}^{2}+v_{1}^{2}\right) \mathrm{d} x \mathrm{~d} y=-\frac{R e}{L_{x}} \int_{0}^{1} \int_{0}^{L_{x}} u_{0 y} u_{1} v_{1} \mathrm{~d} x \mathrm{~d} y \\
& -\left.\frac{1}{L_{x}} \int_{0}^{L_{x}} v_{1}\left[\left(\left|\hat{\tau}_{w 0}\right| \nu P_{w 1}(k / \nu)+2 \cot \theta\right) \eta-R e W e \eta_{x x}-2 \hat{\tau}_{w 0} \eta_{x x}-2 u_{1 x}\right]\right|_{y=1} \mathrm{~d} x \\
& \quad+\frac{1}{L_{x}} \int_{0}^{1} \int_{0}^{L_{x}}\left(u_{1} u_{1 x x}+u_{1} u_{1 y y}+v_{1} v_{1 x x}+v_{1} v_{1 y y}\right) \mathrm{d} x \mathrm{~d} y
\end{aligned}
$$

The above equation can be further simplified by substituting the square of the continuity equation (5.10). This yields

$$
E_{K}=E_{S T}+E_{G P}+E_{R}+E_{S H}+E_{D}+E_{T 1}
$$

where

$$
E_{K}=\frac{1}{2 L_{x}} \frac{d}{d t} \int_{0}^{1} \int_{0}^{L_{x}}\left(u_{1}^{2}+v_{1}^{2}\right) \mathrm{d} x \mathrm{~d} y
$$


is the rate of change of "kinetic energy" of the disturbance, which is proportional to the growth rate. The disturbance energy production due to surface tension forces is given by

$$
E_{S T}=\left.\frac{W e}{L_{x}} \int_{0}^{L_{x}} v_{1}\right|_{1} \eta_{x x} \mathrm{~d} x .
$$

The rate of work done by the pressure gradient (due to gas flow and gravity) in deforming the interface is given by

$$
E_{G P}=-\left.\frac{1}{L_{x} R e} \int_{0}^{L_{x}} v_{1}\right|_{1}\left[\left|\hat{\tau}_{w 0}\right| \nu P_{w 1}(k / \nu)+2 \cot \theta\right] \eta \mathrm{d} x,
$$

whereas the term $E_{R}$ corresponds to the rate of energy transfer from the mean flow of the liquid to the disturbance by means of Reynolds stresses, and is given by

$$
E_{R}=-\frac{1}{L_{x}} \int_{0}^{1} \int_{0}^{L_{x}} u_{0 y} u_{1} v_{1} \mathrm{~d} x \mathrm{~d} y .
$$

The work done by the disturbance shear force at the interface is represented by

$$
E_{S H}=\left.\frac{1}{L_{x} R e} \int_{0}^{L_{x}} u_{1}\right|_{1}\left[u_{1 y}+v_{1 x}\right]_{1} \mathrm{~d} x
$$

whereas, the rate of dissipation of energy by viscous forces is given by

$$
E_{D}=-\frac{1}{L_{x} R e} \int_{0}^{1} \int_{0}^{L_{x}}\left[2\left(u_{1 x}\right)^{2}+\left(u_{1 y}+v_{1 x}\right)^{2}+2\left(v_{1 y}\right)^{2}\right] \mathrm{d} x \mathrm{~d} y .
$$

Finally, the work done by the applied shear stress at the interface due to countercurrent gas flow is given by

$$
E_{T 1}=\left.\frac{2 \hat{\tau}_{w 0}}{L_{x} R e} \int_{0}^{L_{x}} v_{1}\right|_{1} \eta_{x} \mathrm{~d} x .
$$

The various energy quantities (5.18)-(5.24) in the kinetic energy equation (5.17) can be evaluated by making use of the solution obtained by solving the eigenvalue problem (A 7). The variables involved in the energy terms are derived once the eigenvalue $c$ and the eigenfunction $\phi$ are obtained by solving the OS problem. In order to perform the integration along the cross-streamwise direction, we employ a pseudo-spectral approach, whereas integration along the streamwise direction is carried out using the trapezoidal rule.

In this energy analysis, we are interested in the energy composition for the most dominant normal mode, therefore we consider wavenumbers corresponding to the maximum growthrate. The various energy terms in the kinetic energy equation are evaluated for increasing values of gas flow rate $\Theta$ using the numerical methodology described above, and the results, scaled with the kinetic energy $E_{K}$, are shown in figure 13 . For the case of a vertically falling film in the absence of gas flow $(\Theta=0)$, the main contribution to energy production comes from the disturbance shear force at the interface $\widehat{E}_{S H}$ whereas some of this energy is dissipated by the viscous forces $\widehat{E}_{D}$. As the gas flow is switched on, the energy production has a contribution from the applied shear stress $\widehat{E}_{T 1}$ in addition to the applied pressure gradient due to gas flow and gravity $\widehat{E}_{G P}$. (We note that for the vertical configuration of the channel considered here, the contribution due to gravity in $\widehat{E}_{G P}$ vanishes.) It can be observed that $\widehat{E}_{T 1}$ dominates over $\widehat{E}_{G P}$ for smaller values of the gas flow rate, whereas $\widehat{E}_{G P}$ is dominant for larger gas flow rates. The contribution from the Reynolds stresses $\widehat{E}_{R}$ is negligible for the moderate values of the Reynolds number considered in this study. 


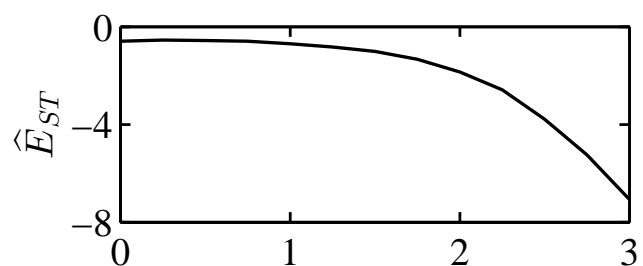

$\Theta$
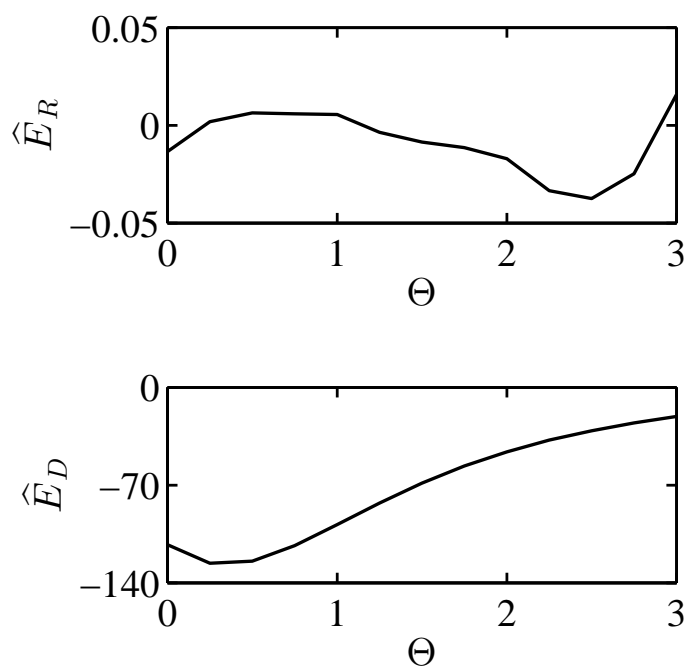

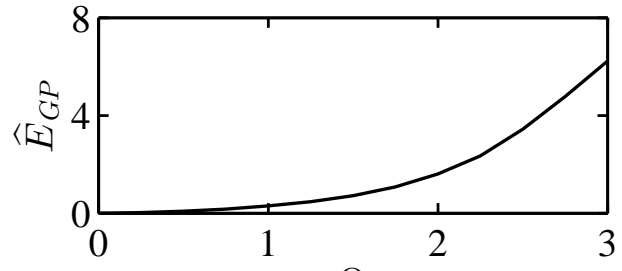

$\Theta$

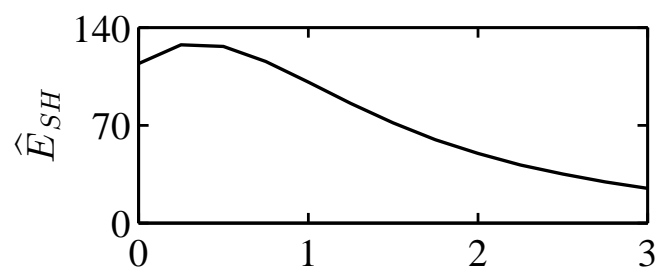

$\Theta$

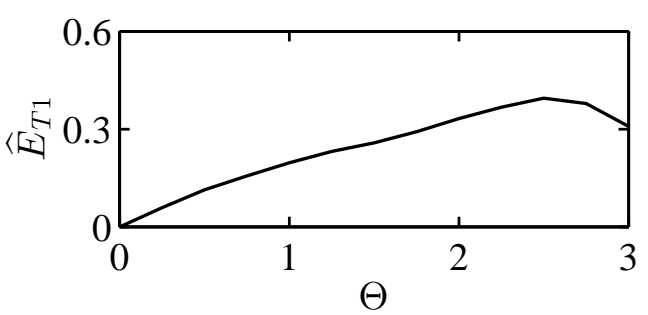

FIGURE 13. Plots of various energy terms in equation (5.17) normalised with the total kinetic energy $E_{K}$ as a function of gas shear $\Theta$, when $R e_{0}=10, \theta=\pi / 2, K a=1988.5$ and $N=0.4$.

\subsection{Absolute and convective instabilities}

In this section, we outline our methodology to analyse absolute and convective instabilities by considering the dispersion relation obtained from the long-wave model. Our analysis follows that by Fokas \& Papageorgiou (2005). We note first that the dispersion relation $s(k)$ is not analytic at zero and, therefore, it is not possible to study absolute and convective instabilities in a straightforward way by analysing the saddle point of the real part of the dispersion relation and applying the Briggs pinching criterion, see e.g. Huerre \& Monkewitz (1990). To begin, let us assume that $f(x, 0)=f_{0}(x)$ is the given initial condition. Therefore, the solution of the linearised equation can be written as

$$
f(x, t)=\frac{1}{2 \pi} \int_{-\infty}^{\infty} \mathrm{e}^{s(k) t+\mathrm{i} k x} \hat{f}_{0}(k) \mathrm{d} k
$$

where $\hat{f}_{0}(k)$ is the Fourier transform of the initial condition:

$$
\hat{f}_{0}(k)=\int_{-\infty}^{\infty} \mathrm{e}^{-\mathrm{i} k x} f_{0}(x) \mathrm{d} x .
$$

There are two possibilities: if $\operatorname{Re}[s(k)] \leqslant 0$ for all $k$, the flat-film solution is linearly stable. On the other hand, if $\operatorname{Re}[s(k)]>0$ at least for some $k$, then the flat-film solution is unstable, and the instability could be either absolute or convective. Noting that $\tau_{w 1}(-\alpha)=\overline{\tau_{w 1}(\alpha)}$ for real $\alpha$, where the bar denotes complex conjugation (see Vellingiri 
et al. 2013), the growth rate can be written as

$$
s(k)= \begin{cases}-\mathrm{i} a k+b k^{2}-c k^{4}-\mathrm{i} d k \overline{\tau_{w 1}(k / \nu)} \equiv s_{-}(k), & \text { if } k<0, \\ -\mathrm{i} a k+b k^{2}-c k^{4}-\mathrm{i} d k \tau_{w 1}(k / \nu) \equiv s_{+}(k), & \text { if } k \geqslant 0 .\end{cases}
$$

The growth rates are related by $s_{-}(-k)=\overline{s_{+}(k)}$. We denote the real part of the growth rate as $s_{r}(k) \equiv \operatorname{Re}\left[s_{+}(k)\right]=b k^{2}-c k^{4}+d k \operatorname{Im}\left[\tau_{w 1}(k / \nu)\right]$. The integral (5.25) can be rewritten as

$$
f(x, t)=\frac{1}{2 \pi}[I(x, t)+J(x, t)]
$$

where

$$
I(x, t)=\left(\int_{-\infty}^{-k_{1}}+\int_{k_{1}}^{\infty}\right) \mathrm{e}^{s(k) t+\mathrm{i} k x} \hat{f}_{0}(k) \mathrm{d} k
$$

and

$$
J(x, t)=\left(\int_{-k_{1}}^{0}+\int_{0}^{k_{1}}\right) \mathrm{e}^{s(k) t+\mathrm{i} k x} \hat{f}_{0}(k) \mathrm{d} k,
$$

with $k_{1}$ being the critical wavenumber below which the flat film solution is unstable. For fixed $x / t, I(x, t)$ goes to zero as $t$ increases. Therefore, it is sufficient to analyse the other integral, $J(x, t)$ :

$$
\begin{aligned}
J(x, t) & =\int_{-k_{1}}^{0} \mathrm{e}^{s_{-}(k) t+\mathrm{i} k x} \hat{f}_{0}(k) \mathrm{d} k+\int_{0}^{k_{1}} \mathrm{e}^{s_{+}(k) t+\mathrm{i} k x} \hat{f}_{0}(k) \mathrm{d} k \\
& =\int_{0}^{k_{1}} \mathrm{e}^{s_{-}(-k) t-\mathrm{i} k x} \hat{f}_{0}(-k) \mathrm{d} k+\int_{0}^{k_{1}} \mathrm{e}^{s_{+}(k) t+\mathrm{i} k x} \hat{f}_{0}(k) \mathrm{d} k .
\end{aligned}
$$

By making use of the fact that $s_{-}(-k)=\overline{s_{+}(k)}$ for real $k$, and that $\hat{f}_{0}(-k)=\overline{\hat{f}_{0}(k)}$ as $f_{0}(x)$ is a real function, we obtain

$$
\begin{aligned}
J(x, t) & =\int_{0}^{k_{1}}\left[\mathrm{e}^{s_{+}(k) t-\mathrm{i} k x} \overline{\hat{f}_{0}(k)}+\mathrm{e}^{s_{+}(k) t+\mathrm{i} k x} \hat{f}_{0}(k)\right] \mathrm{d} k \\
& =\int_{0}^{k_{1}}\left[\overline{\mathrm{e}^{s_{+}(k) t+\mathrm{i} k x} \hat{f}_{0}(k)}+\mathrm{e}^{s_{+}(k) t+\mathrm{i} k x} \hat{f}_{0}(k)\right] \mathrm{d} k \\
& =2 \operatorname{Re}[K(x, t)]
\end{aligned}
$$

where

$$
K(x, t)=\int_{0}^{k_{1}} \mathrm{e}^{s_{+}(k) t+\mathrm{i} k x} \hat{f}_{0}(k) \mathrm{d} k .
$$

The function $s_{+}(k)$ has been defined for real non-negative values of $k$, since $\tau_{w 1}(\alpha)$ is obtained by solving equation (3.34) with homogeneous boundary conditions (3.37) and (3.38) in Tseluiko \& Kalliadasis (2011) for positive values of $\alpha$. Noticing that the coefficients in (3.34) in Tseluiko \& Kalliadasis (2011) are analytic functions of $\alpha$, we can obtain an analytic continuation of $\tau_{w 1}(\alpha)$ from the non-negative real semi-axis to the whole complex plane by solving equation (3.34) with homogeneous boundary conditions (3.37) and (3.38) in Tseluiko \& Kalliadasis (2011) when $\alpha$ is an arbitrary complex number. Then the analysis of $K(x, t)$ depends on the topology of the curves $s_{r}(k)=0$ in the complex $k$-plane, where $s_{r}(k)$ denotes the real part of $s_{+}(k)$. There are two possibilities:

Case I: There is part of the curve $s_{r}(k)=0$ that connects the origin $(0,0)$ and $\left(k_{1}, 0\right)$ in the $k_{r} k_{i}$-plane, with $k_{r}$ and $k_{i}$ denoting the real and the imaginary parts of $k$, respectively. In addition, if $\hat{f}_{0}(k)$ can be analytically continued off the real axis so that 


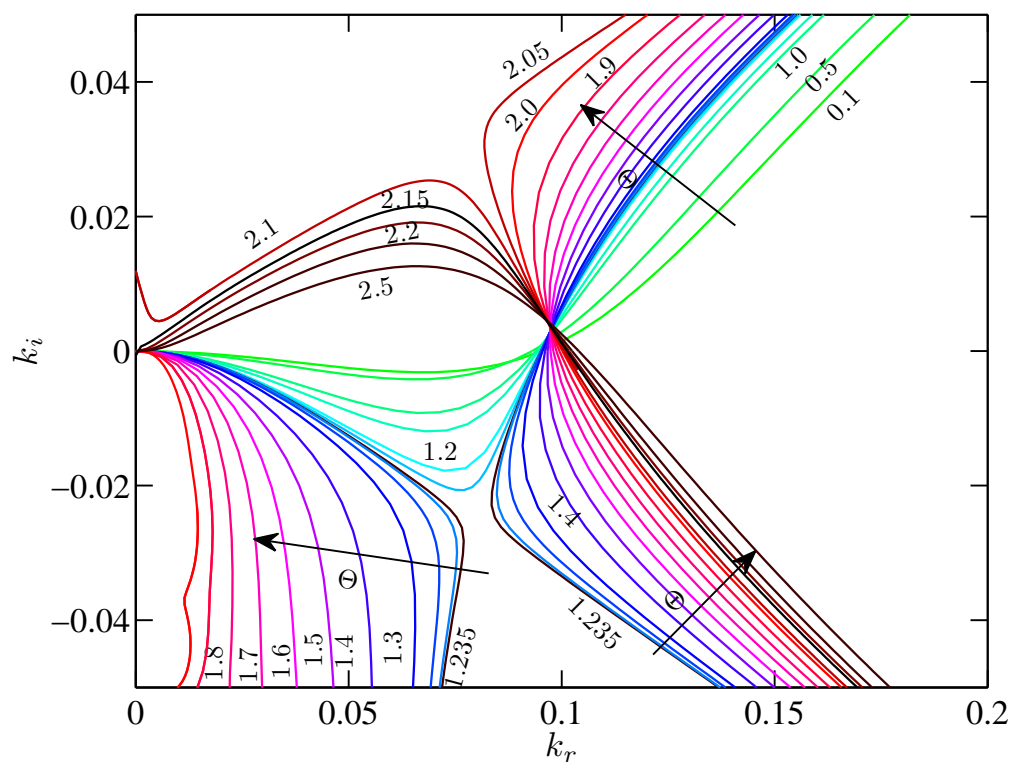

Figure 14. (Color online) The curves of $s_{r}(k)=0$ in the complex wavenumber plane $\left(k_{r}, k_{i}\right)$ from the long-wave model for various values of the gas shear, $\Theta$, when $R e_{0}=10, \theta=\pi / 2$, $K a=1988.5$ and $N=0.4$.

$\hat{f}_{0}(k)$ is analytic in a neighbourhood of the curve $s_{r}(k)=0$ connecting the points $(0,0)$ and $\left(k_{1}, 0\right)$ (this is the case, for example, when $f_{0}(x)$ has a sufficiently fast exponential decay as $|x| \rightarrow \infty)$, the integration contour for $K(x, t)$ can be deformed to follow that part of the curve $s_{r}(k)=0$ which connects $(0,0)$ to $\left(k_{1}, 0\right)$. The Riemann-Lebesgue lemma then implies that for a fixed $x, K(x, t) \rightarrow 0$ as $t \rightarrow \infty$, and we have convective instability.

Case II: The other possibility is that it is impossible to connect the origin with $\left(k_{1}, 0\right)$ using part of the curve $s_{r}(k)=0$ in the $k_{r} k_{i}$-plane. In such a scenario, there must exist a saddle point (also called the pinch point) of $s_{r}(k)$, at which $s_{r}(k)$ is positive, so that the integration contour can be deformed into a steepest descent path passing through this saddle point. Here $K(x, t)$ is seen to grow for a fixed $x$ as $t \rightarrow \infty$ (Hinch 1991), and the type of instability in this case is absolute. We also note here that the saddle point corresponds to the mode with zero group velocity.

The details of the numerical scheme to compute the curves $s_{r}(k)=0$ in the $k_{r} k_{i^{-}}$ plane for the OS problem is provided in Appendix C. We begin by presenting in figure 14 the curves $s_{r}(k)=0$ in the $\left(k_{r}, k_{i}\right)$ plane for various values of the gas shear, $\Theta$ for $R e=5$, corresponding to the dispersion relation (4.15) obtained from the long-wave model. It can be observed from figure 14 that for sufficiently small values of $\Theta$, the origin is connected to $\left(k_{r}, 0\right)$. Therefore, we have convective instability. This is to be expected, as any localised disturbance introduced on the system is convected downstream, in the positive $x$-direction. The situation is similar for large values of $\Theta$, but the disturbance is convected in the negative $x$-direction. However, there exists a band of gas flow rates $(1.235 \leqslant \Theta \leqslant 2.1)$ for which the origin is not connected to $\left(k_{r}, 0\right)$ by $s_{r}(k)=0$, and the instability is absolute.

Figure 15 shows the curves $s_{r}(k)=0$ corresponding to the quadratic dispersion relation (4.18) obtained from the WIBL model. We note that for the WIBL model there exist 


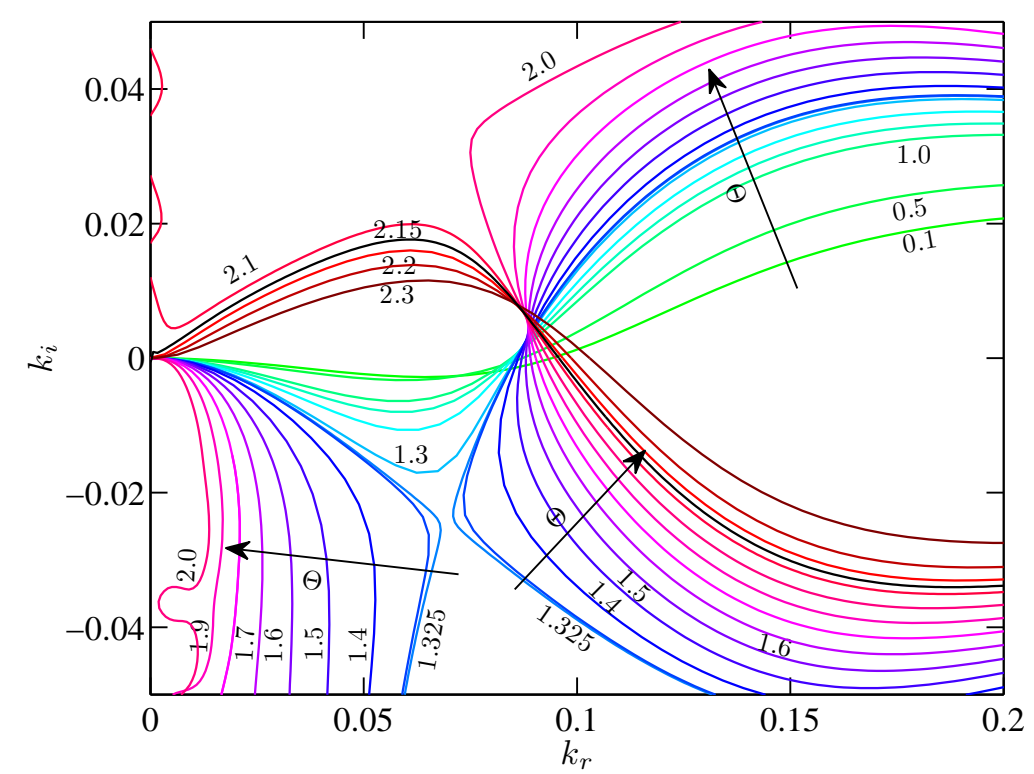

Figure 15. (Color online) The curves of $s_{r}(k)=0$ in the complex wavenumber plane $\left(k_{r}, k_{i}\right)$ from the WIBL model for various values of the gas shear, $\Theta$, when $R e_{0}=10, \theta=\pi / 2$, $K a=1988.5$ and $N=0.4$.

two branches, $s_{r 1}(k)$ and $s_{r 2}(k)$. However, for the parameter space under consideration, only for one of these branches there exists a band of unstable wave numbers. We denote this branch by $s_{r}(k)$. The results obtained using the WIBL model are similar to those obtained from the long-wave model with only minor differences.

Finally, we present the curves $s_{r}(k)=0$, in the $\left(k_{r}, k_{i}\right)$ plane corresponding to the dominant mode in the OS problem (figure 16), which also turns out to be the only unstable mode for the parameter values considered. The results are again similar to those presented earlier for the long-wave and WIBL models, with the region of absolute instability predicted to be $1.21 \leqslant \Theta \leqslant 2.1$. This is not very surprising as the liquid flow rate that we have considered is sufficiently small for the low-dimensional models to have good agreement with the OS results (see also figure 12, from the temporal analysis). Moreover, we find that the WIBL model has a better quantitative agreement with the OS equations than the long-wave model, in the flow regime considered.

We note in addition, the non-linear computations of the WIBL model of Tseluiko \& Kalliadasis (2011) reveal that the flooding point (defined as the point at which there appear large-amplitude nearly stationary waves) is close to $\Theta \sim 2.5$. Interestingly, this flooding point is also close to the upper limit of the absolute instability regime $(\Theta \sim 2.1)$ predicted by our analysis.

\subsubsection{Influence of the inclination angle}

We analyse the influence of the inclination angle on the onset of absolute and convective instabilities by performing the analysis for the OS problem for various values of inclination angle $\theta$, namely $\pi / 3, \pi / 4$ and $\pi / 6$, with the modified Reynolds number being fixed at $R e_{0}=10$. Table 1 shows regimes of absolute and convective instabilities for the various angles (the results for $\pi / 2$ are shown for the various models, whereas for the other angles the results are obtained by performing the analysis for the OS problem only). Note that 


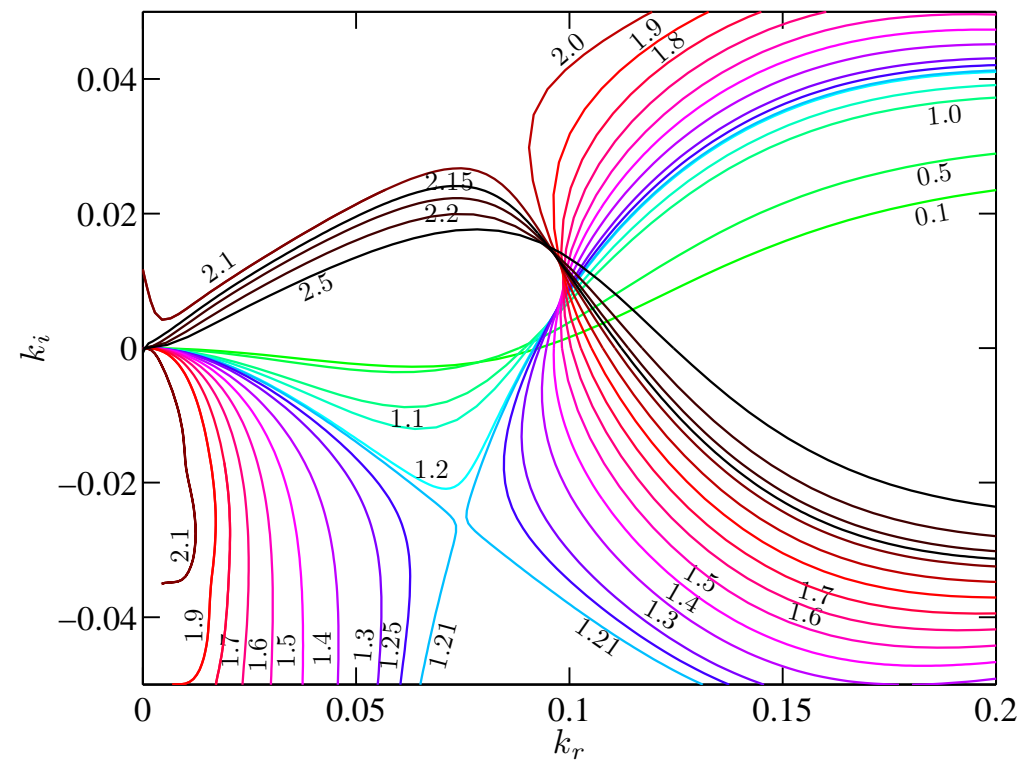

Figure 16. (Color online) The curves of $s_{r}(k)=0$ in the complex wavenumber plane $\left(k_{r}, k_{i}\right)$ by solving the OS problem for various values of the gas shear, $\Theta$, when $R e_{0}=10, \theta=\pi / 2$, $K a=1988.5$ and $N=0.4$.

$\begin{array}{lccc}\text { Model } & \text { angle } & \text { absolute instability } & \text { convective instability } \\ \text { LW } & \pi / 2 & 1.235 \leqslant \Theta \leqslant 2.1 & 0<\Theta<1.235, \Theta>2.1 \\ \text { WIBL } & \pi / 2 & 1.325 \leqslant \Theta \leqslant 2.1 & 0<\Theta<1.325, \Theta>2.1 \\ \text { OS } & \pi / 2 & 1.21 \leqslant \Theta \leqslant 2.1 & 0<\Theta<1.21, \Theta>2.1 \\ & \pi / 3 & 1.2 \leqslant \Theta \leqslant 1.8 & 0<\Theta<1.2, \Theta>1.8 \\ & \pi / 4 & 1.0 \leqslant \Theta \leqslant 1.5 & 0<\Theta<1.0, \Theta>1.5 \\ & \pi / 6 & 0.8 \leqslant \Theta \leqslant 1.0 & 0<\Theta<0.8, \Theta>1.0\end{array}$

TABLE 1. Absolute and convective instability regimes computed using various models when $R e_{0}=10, K a=1988.5$ and $N=0.4$.

as the inclination angle of the channel is decreased, the liquid flow rate decreases as well and the stabilisation of the interface is enhanced by the normal component of gravity resulting from the decrease of the inclination angle. It can be observed that the increase in the inclination angle results in delaying the onset of absolute instability.

\subsubsection{Comparison with experiments}

In order to verify the predictions of our model, we consider air and methanol as working fluids in a vertical channel that were also considered in the experiments by Zapke \& Kröger (2000a) (see their figure 8). As the parameters fall in the regime where our WIBL model was shown to have a good agreement with the OS problem, we consider the WIBL model to compute the limits of absolute instability, which we believe has a close connection to flooding. The comparison shown in table 2 indicates a fair agreement. The experimental flooding point turns out to be close to the region of absolute instability (moreover, it turns out that the flooding point lies between the absolute instability boundaries, except for the last line in the table), but it has to be noted that the trend is slightly different, i.e. gas velocities for the absolute instability boundaries monotonically 


$\begin{array}{lccc}V_{\ell}(\mathrm{m} / \mathrm{s}) & V_{g, Z K}(\mathrm{~m} / \mathrm{s}) & V_{g, \text { low }}(\mathrm{m} / \mathrm{s}) & V_{g, \text { up }}(\mathrm{m} / \mathrm{s}) \\ 0.011 & 7.96 & 6.46 & 8.08 \\ 0.019 & 7.31 & 6.95 & 9.24 \\ 0.044 & 6.25 & 7.33 & 11.21 \\ 0.055 & 5.57 & 7.35 & 11.86\end{array}$

TABLE 2. Comparison with experiments of Zapke \& Kröger (2000a) with air and methanol as working fluids (see their figure 8). $V_{\ell}$ denotes the average liquid velocity, $V_{g, Z K}$ corresponds to the average flooding gas velocity in Zapke \& Kröger $(2000 a), V_{g, l o w}$ and $V_{g}$,up corresponds to the lower and upper limits of absolute instability, respectively, computed using our analysis.

increase as the liquid velocity increases, whereas in the experiments of Zapke \& Kröger $(2000 a)$ the flooding gas velocity is found to decrease with the liquid velocity (which appears to be counterintuitive). However, confidence limits-error bars were not provided for the experiments of Zapke \& Kröger (2000a), and, therefore, it is not really clear how accurate are the experimental findings. Another explanation for the differences between our theoretical predictions and the experiments may be that our analysis assumes two-dimensional flow whereas two-dimensionality may not be valid in the experiments. Moreover, the observed differences may be related to the way Zapke \& Kröger (2000a) define flooding and their interpretation of flooding, namely they state that flooding occurs when a vortex (large amplitude wave) formed at the inlet of the channel has grown to an extent that it obstructs the flow of the gas (i.e. the height of the wave is comparable to the height of the channel) resulting in the liquid travelling up past the injection point. (We note that Zapke \& Kröger (2000a) used a channel with a rectangular cross section of width $10 \mathrm{~mm}$ and height $50 \mathrm{~mm}$.) But the width of the channel considered in our model is assumed to be very large compared to the liquid film thickness so that the amplitude of the standing wave formed at the point of wave reversal is not comparable to the channel width. Notwithstanding the above mentioned disparities, our linear theory appears to be useful in predicting the nonlinear phenomenon of flooding, but clearly further detailed experiments are needed to test carefully our theoretical predictions.

\subsection{Time-dependent computations}

We further explore the absolute and convective instability regimes predicted by our linear analysis in $\$ 5.3$, by solving the linearised WIBL model (4.16)-(4.17) as an initial-value problem subject to a localised initial condition. This is carried out numerically using a scheme which is based on a Fourier pseudo-spectral representation of the derivatives along the spatial direction and an adaptive stepping along time, e.g. Tseluiko et al. (2010). We choose the initial condition of the interface disturbance, $f(x, t)$, to be localised about $x=0$, namely, we choose the Gaussian function $f(x, 0)=0.1 \exp \left(-0.5 x^{2}\right)$. In addition we choose $g(x, 0)=0$. The liquid Reynolds number is taken to be $R e=5$ consistent with that in the previous section. The equations are integrated on a long periodic domain, $[-750,750]$, for sufficiently long time, say $t \approx 500$, to determine the evolution of the wave packets.

We begin with a relatively small value of the gas shear, $\Theta=1.0$, and the corresponding evolution of the disturbance is depicted as a space-time plot in figure 17a. It can be observed that the wave packet starts propagating downstream along the positive $x$-direction, therefore the instability is convective. On the other side of the gas flow spectrum, e.g. for $\Theta=2.2$ the instability is again convective with the wave packet propagating upstream along the negative $x$-direction (figure $17 \mathrm{~b}$ ). This can further be confirmed by 
(a)

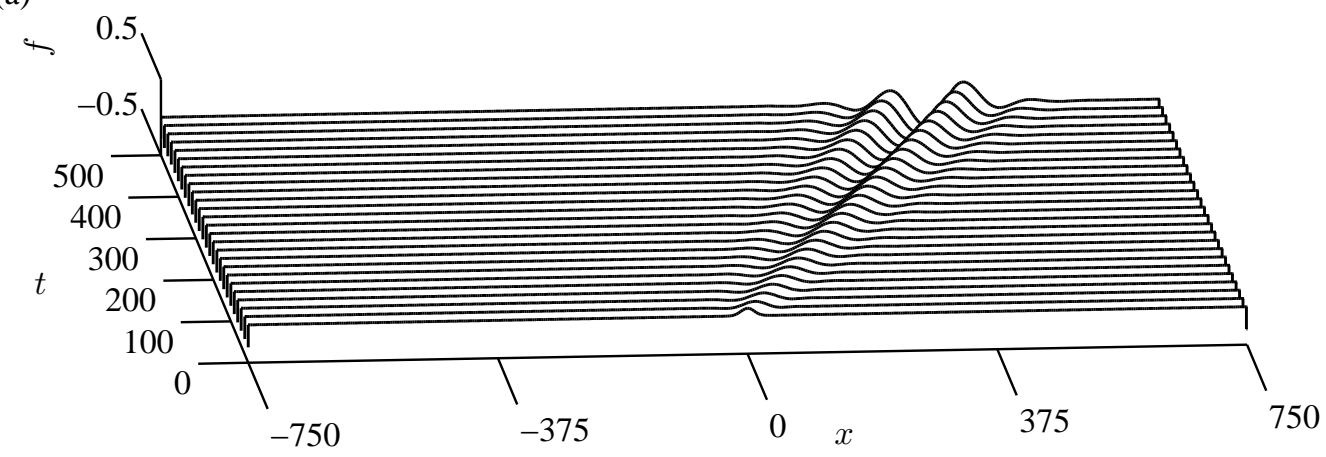

(b)

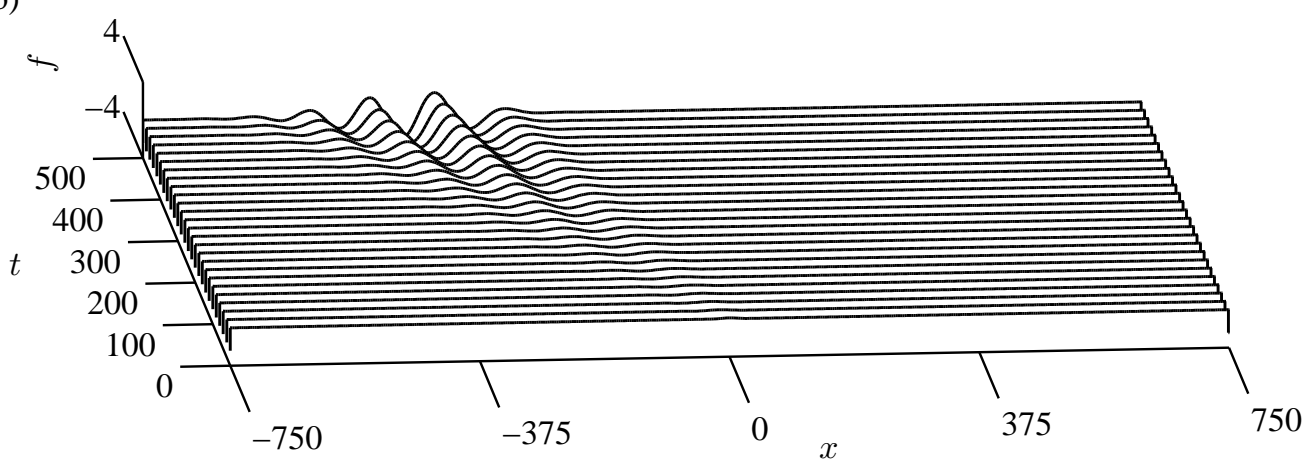

FIgURE 17. Time-dependent computation of the linearised WIBL model subject to a localised disturbance, showing convective instability for (a) $\Theta=1.0$ and (b) $\Theta=2.2$ with $R e_{0}=10$, $\theta=\pi / 2, K a=1988.5$ and $N=0.4$. The three-dimensional plot is inclined here for better visualisation.

examining the wave profile at a sufficiently long time instant and the time evolution of the disturbance, $f(x, t)$ at $x=0$, see top and bottom panels of figure 18, respectively. From figure 18, it can be ascertained that the wave profiles for $\Theta=1.0$ and $\Theta=2.2$ are convected in the positive and negative $x$-direction (see the top panels), and also the disturbance, $f(0, t)$ eventually decays to zero (see the bottom panels). This is consistent with the analytical results discussed above. For intermediate values of gas flow rates, e.g. for $\Theta=1.5$ and $\Theta=1.65$ shown in figure 19, the wave packets invade both the negative and the positive $x$-directions, and for longer times they are expected to contaminate the whole domain. This can also be established from figure 20, from which it can be concluded that the disturbance $f(0, t)$ does not decay to zero. These results agree with the band of absolutely unstable gas flow rates, $1.2 \leqslant \Theta \leqslant 2.1$ predicted by our analysis of various models in $\S 5.3$.

\section{Concluding remarks}

We have analysed the linear stability of a gas-liquid system in an inclined channel, where a thin liquid film flowing along the bottom wall is in contact with a countercurrent gas flow occupying the rest of the channel. The liquid film is considered to be laminar whereas the counter-current gas flow is turbulent. The influence of gas flow enters the liquid problem through the boundary conditions at the gas-liquid interface as 
(a)
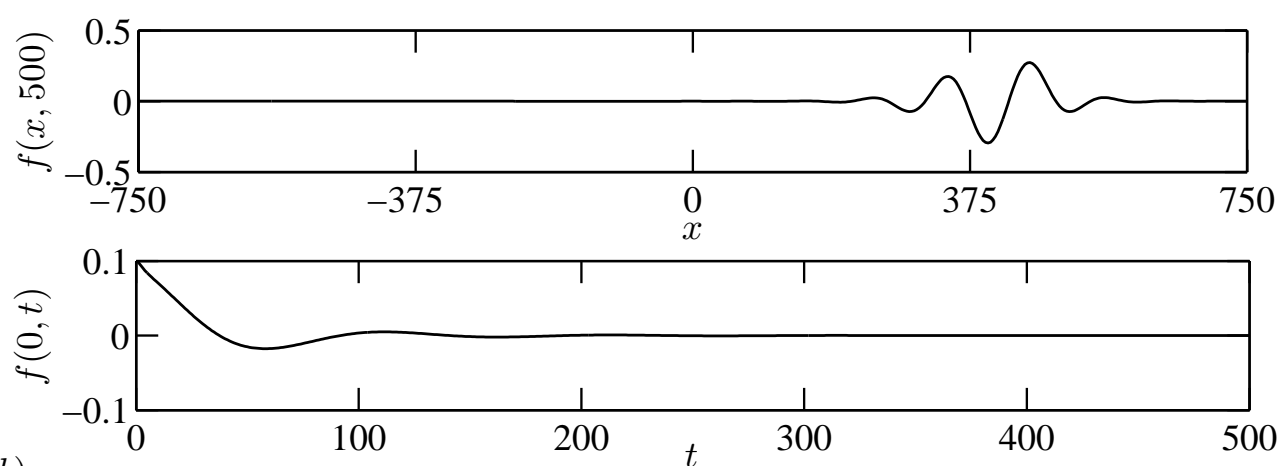

(b)
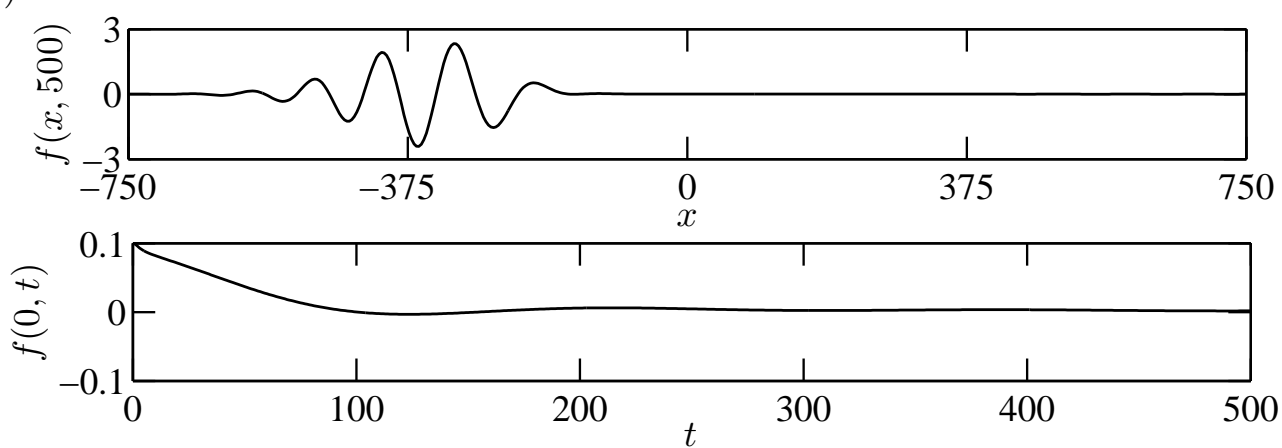

FiguRE 18. Time-dependent computations of the linearised WIBL model depicting wave profiles at $t=500$ and time evolution of the disturbance at $x=0$ for (a) $\Theta=1.0$ and (b) $\Theta=2.2$ with $R e_{0}=10, \theta=\pi / 2, K a=1988.5$ and $N=0.4$.

expected. This challenging gas-liquid problem can be decoupled and solved independently by making a number of reasonable assumptions (Tseluiko \& Kalliadasis 2011).

We considered two non-local reduced-order model equations for the interface thickness (measured from the liquid layer), namely the long-wave and WIBL models derived by Tseluiko \& Kalliadasis (2011), and also formulated the OS system of equations from the full governing equations and boundary conditions. A significant advantage with the OS equations from a stability perspective is that it is applicable for all values of the liquid Reynolds number and wavenumber. Also, the comparison of the results for the reduced model equations with the OS results is important for finding out the ranges of validity of the reduced models. In addition to performing the temporal stability analysis, we carried out an energy analysis using the linearised version of the full Navier-Stokes equation to understand the physical nature of the instability. We have also developed a generalised methodology based on Fokas \& Papageorgiou (2005) for the non-local equations with non-analytic dispersion relations to scrutinise absolute and convective instabilities. This approach involves analysing the nature of growth-rate curves in the complex wavenumber plane. If the origin is connected to the purely real wavenumber root in the complex wavenumber plane by the vanishing growth rate curve, then the instability is convective. On the other hand, if it is impossible to connect the origin with the real root by the zero-growth-rate curve, and there exists a saddle point (which is also the pinch point) where the growth rate is positive, we have absolute instability. This saddle point also corresponds to the normal mode whose group velocity is zero. 
(a)

(b)
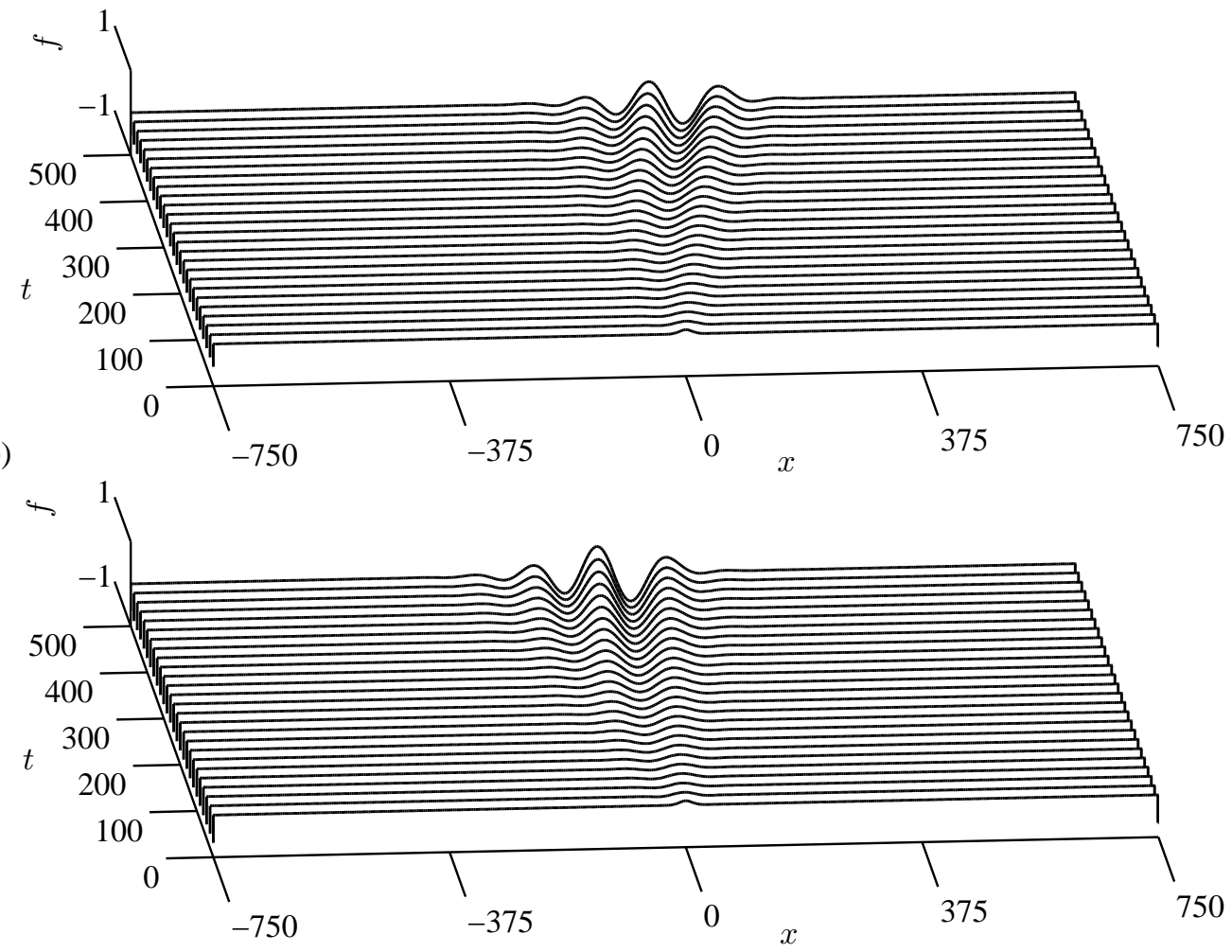

Figure 19. Time-dependent computation of the linearised WIBL model subject to a localised disturbance, showing convective instability for (a) $\Theta=1.5$ and (b) $\Theta=1.65$ with $R e_{0}=10$, $\theta=\pi / 2, K a=1988.5$ and $N=0.4$. The three-dimensional plot is inclined here for better visualisation.

By applying this methodology to the OS equations, and the low-dimensional models, we obtained the flow conditions for which the system exhibits absolute and convective instabilities. More precisely, we found that for a fixed liquid flow rate, the system is convectively unstable at low and high gas flow rates, where the localised disturbance gets convected in the downstream and upstream directions, respectively. However, for a range of intermediate gas flow rates with the liquid flow rate fixed at the same value as before, we have absolute instability, where the disturbance contaminates the whole domain. We have also analysed the effect of the inclination angle on the onset of absolute instability and we found that larger gas flow rates are required to trigger absolute instability for larger values of the angle of inclination. In general, the results for the WIBL model are found to be in better agreement with the results for the OS equations than the results for the longwave model, as the WIBL model is expected to be able to describe the flow behaviour for up to moderate values of the Reynolds number. Further, we supplemented our analysis by performing time-dependent computations of the linearised WIBL model subject to a localised initial condition, which showed good agreement with the predictions of the linear stability analysis. It is interesting to note that the upper limit of the absolute instability regime is close to the flooding point obtained from the fully non-linear computations of the WIBL model of Tseluiko \& Kalliadasis (2011). In addition, a comparison of our model predictions with the flooding experiments of Zapke \& Kröger (2000a) shows a 
(a)
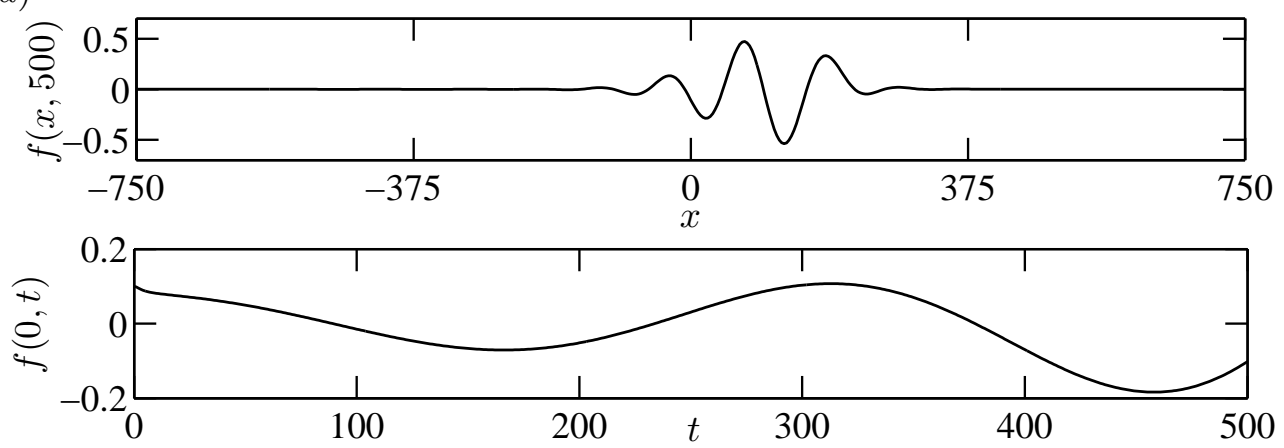

$(b)$
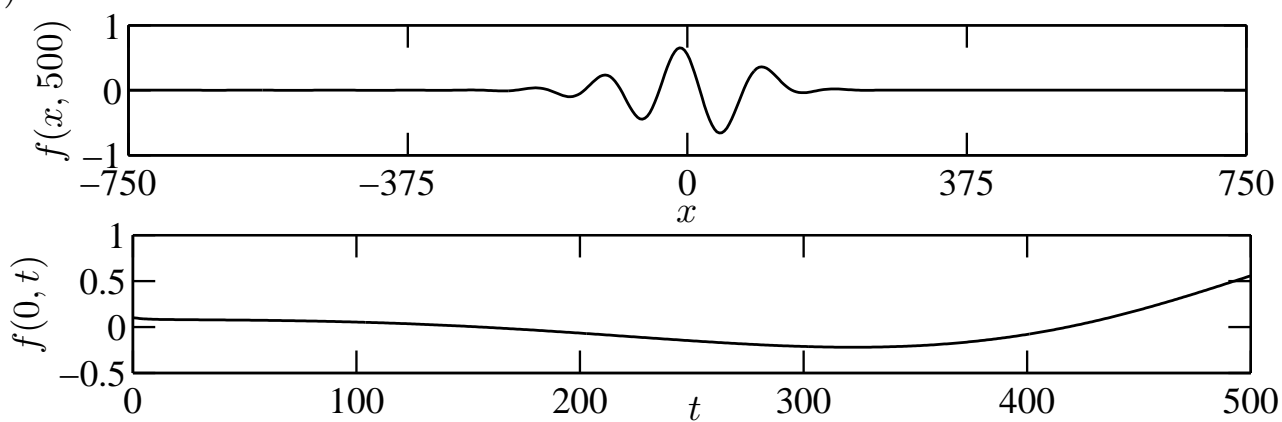

FiguRE 20. Time-dependent computations of the linearised WIBL model depicting wave profiles at $t=500$ and time evolution of the disturbance at $x=0$ for (a) $\Theta=1.5$ and (b) $\Theta=1.65$ with $R e_{0}=10, \theta=\pi / 2, K a=1988.5$ and $N=0.4$.

fairly good agreement. We can therefore conclude that the linear analysis presented here can be useful in predicting the flooding point.

We acknowledge financial support from EPSRC Grant No. EP/K504130/1 and ERC Advanced Grant No. 247031. The work of DT was partly supported by EPSRC Grant No. EP/J001740/1.

\section{Appendix A. Numerical method for the OS problem}

The OS equation (4.8) along with the boundary conditions (4.9)-(4.12) is solved using a Chebyshev pseudo-spectral method (Trefethen 2000; Yatsyshin et al. 2012). By making use of the transformation $z=2 y-1$, we map the physical film domain $0 \leqslant y \leqslant 1$ onto the canonical interval $-1 \leqslant z \leqslant 1$. Under this mapping, the OS equation together with the boundary conditions become

$$
\begin{gathered}
16 \phi^{\prime \prime \prime \prime}-8 k^{2} \phi^{\prime \prime}+k^{4} \phi-\mathrm{i} k \operatorname{Re}\left[\left(\frac{z+1}{4}\right)\left(3+2 \hat{\tau}_{w 0}-z\right)\left(4 \phi^{\prime \prime}-k^{2} \phi\right)+2 \phi\right] \\
=-\mathrm{i} k c R e\left(4 \phi^{\prime \prime}-k^{2} \phi\right), \\
\phi(-1)=0, \quad \phi^{\prime}(-1)=0, \\
\phi(1)+\left(1+\hat{\tau}_{w 0}\right) A_{1}=c A_{1}, \\
4 \phi^{\prime \prime}(1)+k^{2} \phi(1)-\left[\left|\hat{\tau}_{w 0}\right| \nu \tau_{w 1}(k / \nu)+2\right] A_{1}=0,
\end{gathered}
$$


Absolute and convective instabilities in counter-current gas-liquid film flows

$$
\begin{aligned}
& 8 \phi^{\prime \prime \prime}(1)-k\left[2 \mathrm{i} \cot \theta+\mathrm{i} k^{2} W e R e+2 k \hat{\tau}_{w 0}+\mathrm{i}\left|\hat{\tau}_{w 0}\right| \nu P_{w 1}(k / \nu)\right] A_{1} \\
& -2 k\left[\mathrm{i} \operatorname{Re}\left(1+\hat{\tau}_{w 0}\right)+3 k\right] \phi^{\prime}(1)+\mathrm{i} k \operatorname{Re} \hat{\tau}_{w 0} \phi(1)=-2 \mathrm{i} k c R e \phi^{\prime}(1),
\end{aligned}
$$

where the prime denotes differentiation with respect to $z$. We could further eliminate $A_{1}$ from the system of equations using (A 4), followed by making the substitution for $A_{1}$ in (A 3) and (A 5). One could then avoid appending the differentiation matrices with zeros and ones in order to solve for $A_{1}$. Nevertheless, we have done all our computations by retaining $A_{1}$ in our model system as a general practice. But the results obtained by both of these approaches are exactly the same.

Boomkamp et al. (1997) developed a collocation method based on Chebyshev polynomials to solve the eigenvalue problem governing the stability of parallel two-phase flow, which is similar to the methodology developed by Orszag (1971) to investigate the stability of Poiseuille's flow. In our approach, we begin by expanding the eigenfunction $\phi(z)$ as a truncated series of Chebyshev polynomials, $T_{i}(z)$ (defined on the interval $[-1,1]$ ),

$$
\phi(z)=\sum_{i=0}^{M} a_{i+1} T_{i}(z),
$$

with $\phi$ given on the Chebyshev points, $z_{j}=\cos \left(\beta_{j}\right), \beta_{j}=(j-1) \pi / M, j=1, \ldots, M+$ 1. In the 'FFT-based' formulation, Chebyshev differentiation is implemented by using the Fast Fourier Transform (Trefethen 2000). The boundary conditions are imposed by replacing the last four rows (corresponding to the highest modes) with five boundary conditions that we have. This results in a generalised matrix eigenvalue problem of the form

$$
A \boldsymbol{w}=c B \boldsymbol{w}
$$

where $\boldsymbol{w}=\left(a_{0}, \ldots, a_{M}, A_{1}\right)^{T}$, and $A$ and $B$ are square matrices of size $M+2$.

In the 'matrix-based' approach, as the name suggests, the derivative of a function is computed by matrix-vector multiplication. The entries of the differentiation matrix are computed by taking the analytical derivative of the interpolating polynomial, followed by evaluation at the Chebyshev points. To enforce the no-slip and no-penetration boundary conditions at the bottom wall, we follow the approach by Trefethen of multiplying the interpolating polynomial by another polynomial (Trefethen 2000), such that both conditions are satisfied implicitly. This approach enables us to impose the bottom-wall boundary conditions accurately, i.e. exactly at the boundary point. This results in a generalised matrix eigenvalue problem as before, but the square matrices $A$ and $B$ now are of size $M+1$.

The generalised eigenvalue problem (A 7) is solved using Matlab. The large entries in $A$ coming from the higher-order derivatives, and the zero rows in $B$ (coming from the bottom-wall boundary conditions) result in spurious and 'infinite' eigenvalues. We ignore such eigenvalues, and consider only the meaningful finite ones for our calculations. Typically 20-30 collocation points are sufficient to accurately capture the behaviour of the system.

\section{Appendix B. Asymptotic solution for the OS problem in the presence of counter-current gas flow}

As in the falling film setting, we perform regular perturbation expansions for the eigenfunction $(\phi)$ and the complex wave velocity $(c)$ for the liquid problem, under the assumption of long-waves $(k \rightarrow 0)$. However, this requires a solution for the gas problem in the long-wave regime. The idea is to make use of the solution obtained by Lin et al. 
(2013) for the gas phase, and proceed with the asymptotics for the liquid problem. By substituting the following long-wave expansions

$$
\begin{aligned}
\phi & =\phi_{0}+\mathrm{i} k \phi_{1}+\cdots, \\
c & =c_{0}+\mathrm{i} k \phi_{1}+\cdots,
\end{aligned}
$$

in equation (A 1) and the boundary conditions (A 2)-(A 5), at the leading order we have

subject to

$$
\begin{gathered}
\phi_{0}^{\prime \prime \prime \prime}=0 \\
\left(2 c_{0}-1-\hat{\tau}_{w 0}\right) \phi_{0}^{\prime \prime}(1)-\phi_{0}(1)=0
\end{gathered}
$$

$$
\phi_{0}(-1)=0, \quad \phi_{0}^{\prime}(-1)=0, \quad \phi_{0}^{\prime \prime \prime}(1)=0 .
$$

As the problem is linear and homogeneous, we set one of the undetermined constants to unity while solving for $\phi_{0}$. As a result, the leading order solution is given by

$$
\begin{gathered}
\phi_{0}=z^{2}+2 z+1, \\
c=2+\hat{\tau}_{w 0} .
\end{gathered}
$$

At $O(k)$, we have

$$
\begin{gathered}
16 \phi_{1}^{\prime \prime \prime \prime}-2 \mathrm{i} R e\left[\left((z-1)\left(2 \hat{\tau}_{w 0}-z+1\right)-2\left(c_{0}-1-\hat{\tau}_{w 0}\right)\right) \phi_{0}^{\prime \prime}+\phi_{0}\right]=0, \\
4\left(c_{0}-1-\hat{\tau}_{w 0}\right) \phi_{1}^{\prime \prime}+4 c_{1} \phi_{0}^{\prime \prime}-2 \phi_{1}(1)+4\left|\hat{\tau}_{w 0}\right| \phi_{0}(1)=0
\end{gathered}
$$

subject to

$$
\begin{gathered}
\phi_{1}(-1)=0, \quad \phi_{1}^{\prime}(-1)=0, \\
16 \phi_{1}^{\prime \prime \prime}(1)-32\left|\hat{\tau}_{w 0}\right| \phi_{0}^{\prime \prime \prime}(1)+4 \mathrm{i} R e\left(c_{0}-1-\hat{\tau}_{w 0}\right) \phi_{0}^{\prime}(1) \\
+2 \mathrm{i} R e\left|\hat{\tau}_{w 0}\right| \phi_{0}(1)-8 \mathrm{i} \cot \theta \phi_{0}^{\prime \prime}(1)=0 .
\end{gathered}
$$

The solution at this order is given by

$$
\begin{gathered}
\phi_{1}=\frac{1}{480} \mathrm{i} \operatorname{Re}\left(2+\hat{\tau}_{w 0}\right) z^{5}-\frac{1}{96} \mathrm{i} \operatorname{Re}\left(2+\hat{\tau}_{w 0}\right) z^{4}-\frac{1}{48} \mathrm{i}\left(3 \operatorname{Re}\left(2+\hat{\tau}_{w 0}\right)\right. \\
+\cot \theta) z^{3}+\frac{1}{480}\left(-\mathrm{i} \operatorname{Re}\left(2+\hat{\tau}_{w 0}\right)+160 \mathrm{i} \cot \theta+480\right) z^{2} \\
+\frac{1}{480}\left(-17 \mathrm{i} \operatorname{Re}\left(2+\hat{\tau}_{w 0}\right)+80 \mathrm{i} \cot \theta+960\right) z+1, \\
c_{1}=\frac{4 \operatorname{Re}}{15}\left(2+\hat{\tau}_{w 0}\right)-\frac{2}{3} \cot \theta .
\end{gathered}
$$

\section{Appendix C. Numerical scheme to compute absolute convective instability regimes with OS}

We provide an outline of the numerical scheme to compute absolute and convective instabilities implemented for the case of the OS problem. We split the matrices $A$ and $B$, and the eigenvector $\boldsymbol{w}$ into their real and imaginary parts as

$$
A=A_{r}+\mathrm{i} A_{i}, \quad B=B_{r}+\mathrm{i} B_{i}, \quad \boldsymbol{w}=\boldsymbol{w}_{r}+\mathrm{i} \boldsymbol{w}_{i} .
$$

Now, the matrix eigenvalue problem can be written in terms of two real equations, for any given $R e$ and $\Theta$ :

$$
A_{r}\left(k_{r}, k_{i}\right) \boldsymbol{w}_{r}-A_{i}\left(k_{r}, k_{i}\right) \boldsymbol{w}_{i}=c_{r}\left[B_{r}\left(k_{r}, k_{i}\right) \boldsymbol{w}_{r}-B_{i}\left(k_{r}, k_{i}\right) \boldsymbol{w}_{i}\right],
$$




$$
\begin{gathered}
A_{r}\left(k_{r}, k_{i}\right) \boldsymbol{w}_{i}+A_{i}\left(k_{r}, k_{i}\right) \boldsymbol{w}_{r}=c_{r}\left[A_{r}\left(k_{r}, k_{i}\right) \boldsymbol{w}_{i}+A_{i}\left(k_{r}, k_{i}\right) \boldsymbol{w}_{r}\right], \\
k_{r} c_{i}+k_{i} c_{r}=0 .
\end{gathered}
$$

The last equation $(\mathrm{C} 2 c)$ comes from the requirement that the real part of the growth rate is zero, i.e. $s_{r}\left(k_{r}, k_{i}\right)=0$. So far, we have $2 M+3$ equations for $2 M+6$ unknowns in $k_{r}, k_{i}, \boldsymbol{w}_{r}, \boldsymbol{w}_{i}, c_{r}$ and $c_{i}$. To fix the non-uniqueness of the eigenvector (which follows from the fact that an eigenvector multiplied by any complex number is also an eigenvector) we impose the following conditions:

$$
\boldsymbol{w}_{r}^{T} \boldsymbol{w}_{r}+\boldsymbol{w}_{i}^{T} \boldsymbol{w}_{i}=1, \quad \boldsymbol{w}_{r}^{T} \boldsymbol{w}_{i}=0 .
$$

Finally, we look for solutions at a small distance $\varepsilon_{k}$ from the previous point $\left(k_{r_{0}}, k_{i_{0}}\right)$ on the curve $s_{r}\left(k_{r}, k_{i}\right)=0$. Therefore, we can write

$$
\left(k_{r}-k_{r_{0}}\right)^{2}+\left(k_{i}-k_{i_{0}}\right)^{2}=\varepsilon_{k}^{2} .
$$

The above set of nonlinear equations can be conveniently solved using Newton's method, given an initial solution on $s_{r}\left(k_{r}, k_{i}\right)=0$, which can be obtained by setting $k_{i}=0$. The typical value of $\varepsilon_{k}$ considered is $\sim 10^{-3}$.

\section{REFERENCES}

Alekseenko, S. V., Nakoryakov, V. E. \& Pokusaev, B. G. 1994 Wave Flow of Liquid Films. Begell House, New York.

Ali, A., Vafai, K. \& Khaled, A.-R. A. 2003 Comparative study between parallel and counter flow configurations between air and falling film desiccant in the presence of nanoparticle suspensions. Int. J. Energ. Res. 27, 725-745.

Azzopardi, B. J. 2006 Gas-Liquid Flows. Begell House, New York.

Benjamin, T. B 1957 Wave formation in laminar flow down an inclined plane. J. Fluid Mech. 2, 554-574.

Benjamin, T. B 1959 Shearing flow over a wavy boundary. J. Fluid Mech. 6, 161-205.

Benney, DJ 1966 Long waves on liquid films. J. Math. Phys. 45, 150-155.

Boomkamp, P. A. M, Boersma, B. J, Miesen, R. H. M \& Beijnon, G. V 1997 A Chebyshev collocation method for solving two-phase flow stability problems. J. Comput. Phys. 132, 191-200.

Boomkamp, P. A. M \& Miesen, R. H. M. 1996 Classification of instabilities in parallel twophase flow. Intl J. Multiphase Flow 22, 67-88.

Brevdo, L., Laure, P., Dias, F. \& Bridges, T. J. 1999 Linear pulse structure and signalling in a film flow on an inclined plane. J. Fluid Mech. 396, 37-71.

Briggs, R. 1964 Electron-Stream Interaction with Plasmas. MIT Press.

Camassa, R., Forest, M. G., Lee, L., Ogrosky, H. R. \& Olander, J. 2012 Ring waves as a mass transport mechanism in air-driven core-annular flows. Phys. Rev. E 86, 066305.

Cetinbudaklar, A. G. \& Jameson, G. J. 1969 The mechanism of flooding in vertical countercurrent two-phase flow. Chem. Engng Sci. 24, 1669-1680.

Chang, H.-C. \& Demekhin, E. A. 2002 Complex Wave Dynamics on Thin Films. Elsevier.

Chang, H.-C., Demekhin, E. A. \& Kalaidin, E. N. 1995 Interaction dynamics of solitary waves on a falling film. J. Fluid Mech. 294, 123-154.

Chaté, H. \& Manneville, P. 1996 Phase diagram of the two-dimensional complex GinzburgLandau equation. Physica A 224, 348-368.

Clift, R., Pritchard, C. L. \& Nedderman, R. M. 1966 The effect of viscosity on the flooding conditions in wetted wall columns. Chem. Eng. Sci. 21, 87-95.

Craik, A. D. D. 1966 Wind generated waves in thin liquid films. J. Fluid Mech. 26, 369-392.

Demekhin, E. A. 1981 Nonlinear waves in a liquid film entrained by a turbulent gas stream. Fluid Dyn. 16, 188-193.

Dietze, G. F. \& Ruyer-Quil, C. 2013 Wavy liquid films in interaction with a confined laminar gas flow. J. Fluid Mech. 722, 348-393. 
Duprat, C., Ruyer-Quil, C., Kalliadasis, S. \& Giorgiutti-Dauphiné, F. 2007 Absolute and convective instabilities of a viscous film flowing down a vertical fiber. Phys. Rev. Lett. 98, 244502.

Fokas, A. S. \& PApageorgiou, D. T. 2005 Absolute and convective instability for evolution PDEs on the half-line. Stud. Appl. Math. 114, 95-114.

Gaster, M. 1962 A note on the relation between temporally-increasing and spatially-increasing disturbances in hydrodynamic stability. J. Fluid Mech. 14, 222-224.

Hanratty, T. J. \& Engen, J. M. 1957 Interaction between a turbulent air stream and a moving water surface. AIChE $J$ 3, 299-304.

Hinch, E.J. 1991 Perturbation Methods. Cambridge University Press.

Hooper, A. P \& Boyd, W. G. C 1983 Shear-flow instability at the interface between two viscous fluids. J. Fluid Mech. 128, 507-528.

Huerre, P. 2000 Open shear flow instabilities. In Perspectives in Fluid Dynamics: A Collective Introduction to Current Research (ed. G. K. Batchelor, H. K. Moffatt \& M. G. Worster). Cambridge University Press.

Huerre, P. \& Monkewitz, P. A. 1990 Local and global instabilities in spatially developing flows. Annu. Rev. Fluid Mech. 22, 473-537.

Joo, S. W. \& DAvis, S. H. 1992 Instabilities of three-dimensional viscous falling films. J. Fluid Mech. 242, 529-547.

Juniper, M. P. 2006 The effect of confinement on the stability of two-dimensional shear flows. J. Fluid Mech. 565, 171-195.

Kabov, O. A., Lyulin, Yu. V., Marchuk, I. V. \& Zaitsev, D. V. 2007 Locally heated shear-driven liquid films in microchannels and minichannels. Int. J. Heat Fluid Fl. 28, 103-112.

Kalliadasis, S., Ruyer-Quil, C., Scheid, B. \& Velarde, M. G. 2012 Falling Liquid Films, Springer Series on Applied Mathematical Sciences, vol. 176. Springer.

KAPITZA, P. L. 1948 I. Free flow; II. Fluid flow in the presence of continuous gas flow and heat transfer. In Collected Papers of P. L. Kapitza (1965) (ed. D. Ter Haar), pp. 662-689. Pergamon (Oxford).

Kelly, R. E, Goussis, D. A, Lin, S. P \& Hsu, F. K 1989 The mechanism for surface wave instability in film flow down an inclined plane. Phys. Fluids A 1, 819-828.

Lin, S. P. 2003 Breakup of Liquid Sheets and Jets. Cambridge University Press.

Lin, T.-S. \& Kondic, L. 2010 Thin films flowing down inverted substrates: Two dimensional flow. Phys. Fluids 22, 052105.

Lin, T.-S., Tseluiko, D. \& Kalliadasis, S. 2013 Numerical study of a non-local weakly nonlinear model for a liquid film sheared by a turbulent gas. Procedia IUTAM 11, 98-109.

Liu, J. \& Gollub, J. P 1994 Solitary wave dynamics of film flows. Phys. Fluids 6, 1702-1712.

Malamataris, N. A. \& Balakotaiah, V. 2008 Flow structure underneath the large amplitude waves of a vertically falling film. AIChE $J \mathbf{5 4}, 1725-1740$.

Miesen, R. \& Boersma, B. J 1995 Hydrodynamic stability of a sheared liquid film. J. Fluid Mech. 301, 175-202.

Miles, J. W. 1957 On the generation of surface waves by shear flows. J. Fluid Mech. 3, 185-204.

Mouza, A. A., Pantzali, M. N. \& Paras, S. V. 2005 Falling film and flooding phenomena in small diameter vertical tubes: The influence of liquid properties. Chem. Eng. Sci. 60, 4981-4991.

Ó Náraigh, L., Spelt, P. D. M. \& Shaw, S. J. 2013 Absolute linear instability in laminar and turbulent gas-liquid two-layer channel flow. J. Fluid Mech. 714, 58-94.

Ó NÁraigh, L., Spelt, P. D. M. \& ZAKI, T. A. 2011 Turbulent flow over a liquid layer revisited: multi-equation turbulence modelling. J. Fluid Mech. 683, 357-394.

Orszag, S. A 1971 Accurate solution of the Orr-Sommerfeld stability equation. J. Fluid Mech. 50, 689-703.

Pereira, A. \& Kalliadasis, S. 2008 Dynamics of a falling film with solutal Marangoni effect. Phys. Rev. E 78, 036312.

Ruyer-Quil, C. \& Kalliadasis, S. 2012 Wavy regimes of film flow down a fiber. Phys. Rev. E 85, 046302 . 
Ruyer-Quil, C. \& Manneville, P. 1998 Modeling film flows down inclined planes. Eur. Phys. J. B 6, 277-292.

Ruyer-Quil, C. \& Manneville, P. 2000 Improved modeling of flows down inclined planes. Eur. Phys. J. B 15, 357-369.

Ruyer-Quil, C., Scheid, B., Kalliadasis, S., Velarde, M.G. \& Zeytounian, R. Kh. 2005 Thermocapillary long wave in a liquid film flow. Part 1. Low-dimensional formulation. $J$. Fluid Mech. 538, 199-222.

Ruyer-Quil, C., Treveleyan, P., Giorgiutti-Daphiné, Duprat, C. \& Kalliadasis, S. 2008 Modelling film flows down a fibre. J. Fluid Mech. 603, 431-462.

Schlichting, H. 2000 Boundary-Layer Theory. Springer.

Semyonov, P. A. 1944 Flows of thin liquid films. J. Tech. Phys. (in Russian) 14, 427-437.

Shearer, C. J. \& Davidson, J. F. 1965 The investigation of a standing wave due to gas blowing upwards over a liquid film; its relation to flooding in wetted-wall columns. J. Fluid Mech. 22, 321-335.

Sмiтн, M. K. \& Davis, S. H. 1982 The instability of sheared liquid layers. J. Fluid Mech. 121, $187-206$.

Suslov, S. A. 2006 Numerical aspects of searching convective/absolute instability transition. J. Comp. Phys. 212, 188-217.

Thorsness, C. B., Morrisroe, P. E. \& Hanratty, T. J. 1978 A comparison of linear theory with measurements of the variation of shear stress along a solid wave. Chem. Engng Sci. 33, 579-592.

Tobias, S. M., Proctor, M. R. E. \& Knobloch, E. 1998 Convective and absolute instabilities of fluid flows in finite geometry. Physica D 113, 43-72.

Trefethen, L. N. 2000 Spectral Methods in MATLAB. SIAM, Philadelphia.

Trevelyan, P.M.J. \& Kalliadasis, S. $2004 a$ Dynamics of a reactive falling film at large Péclet numbers. II. Nonlinear waves far from criticality: Integral-boundary-layer approximation. Phys. Fluids 16, 3209-3226.

Trevelyan, P.M.J. \& Kalliadasis, S. $2004 b$ Wave dynamics of a thin liquid-film falling down a heated wall. J. Eng. Math. 50, 177-208.

Trevelyan, P.M.J., Scheid, B., Ruyer-Quil, C. \& Kalliadasis, S. 2007 Heated falling films. J. Fluid Mech. 592, 295-334.

Trifonov, YU. YA. 2010 Counter-current gas-liquid wavy film flow between the vertical plates analyzed using the Navier-Stokes equations. AIChE J 56, 1975-1987.

Tseluiko, D. \& Kalliadasis, S. 2011 Nonlinear waves in counter-current gas-liquid film flow. J. Fluid Mech. 673, 19-59.

Tseluiko, D., Saprykin, S., Duprat, C., Giorgiutti-Dauphiné, F. \& Kalliadasis, S. 2010 Pulse dynamics in low-Reynolds-number interfacial hydrodynamics: Experiments and theory. Physica D 239, 2000-2010.

Vellingiri, R., Tseluiko, D., Savva, N. \& Kalliadasis, S. 2013 Dynamics of a liquid film sheared by a co-flowing turbulent gas. Int. J. Multiphase Flow 56, 93-104.

Vlachogiannis, M. \& Bontozoglou, V. 2001 Observations of solitary wave dynamics of film flows. J. Fluid Mech. 435, 191-215.

Woodmansee, D. E. \& Hanratty, T. J. 1969 Mechanism for the removal of droplets from a liquid surface by a parallel air flow. Chem. Engng Sci. 24, 299 - 307.

Yatsyshin, P., Savva, N. \& Kalliadasis, S. 2012 Spectral methods for the equations of classical density-functional theory: Relaxation dynamics of microscopic films. J. Chem. Phys. 136, 124113.

Yıн, C.-S. 1963 Stability of liquid flow down an inclined plane. Phys. Fluids 6, 321.

ZAPke, A. \& Kröger, D.G. $2000 a$ Countercurrent gas-liquid flow in inclined and vertical ducts - I: Flow patterns, pressure drop characteristics and flooding. Int. J. Multiphase Flow 26, $1439-1455$

ZAPKe, A. \& KRÖGER, D.G. $2000 b$ Countercurrent gas-liquid flow in inclined and vertical ducts - II: The validity of the Froude-Ohnesorge number correlation for flooding. Int. J. Multiphase Flow 26, 1457 - 1468. 